\title{
Shape control of hybrid functionally graded plate through smart application of piezoelectric material using simple plate theory
}

\author{
K. M. Bajoria ${ }^{1} \cdot$ S. A. Patare ${ }^{1}$ (D)
}

Received: 19 March 2020 / Accepted: 29 December 2020 / Published online: 25 January 2021

(c) The Author(s) $2021 \quad$ OPEN

\begin{abstract}
The present study takes its inspiration from notable work in the literature related to the flexural analysis of functionally graded material (FGM) plate along with a smart application of piezoelectric material but maintains its novelty in terms of simple approach, an analytical solution with a wide scope of application. Coupling the plate element with piezoelectric smart material can control deflection, vibration thereby increasing the safety, stability, and life of these elements. Plates made up of functionally graded material further enhances the applicability as two different materials are fused. Analysis of such a system is challenging especially for a closed form mathematical solution along with complex boundary conditions. In the present study, it is proposed to develop a simple analytical model for bending analysis of FGM plate coupled with piezoelectric layers. Polynomial based shear deformation function taken from literature is applied to develop a simple mathematical model. A complete flexural analysis is performed for FGM plate to validate the governing simple mathematical model. Through the smart application of piezoelectric material, the deflection of the FGM plate is controlled in as closed loop feedback system. Analytical solution valid over the entire plate domain is obtained incorporating fixed and simple support types of boundary conditions. The initial part of the study details complete mathematical formulation for the plate under consideration, followed by numerical validation in which results of the present model are compared with notable studies in the literature. Lastly, the smart application through shape control of the FGM plate is demonstrated graphically and numerically. The development and application of the discussed mathematical model presented in this study are complete in all aspects of its mathematical form, solution, and numerical validation.
\end{abstract}

Keywords Shape control $\cdot$ piezoelectric $\cdot$ functionally graded $\cdot$ simple plate theory $\cdot$ beam functions

\section{Introduction}

Health monitoring of the structures is the necessity of time due to an increased risk. Elements made up of piezoelectric material gained wide scope as smart material following the increased demand for safety and stability of elements used in engineering structures. The converse application of the piezoelectric effect which is known as actuation can help to control deflection at critical points in the structure. Mechanical strain-induced related to applied electric charge is seen in selected ceramics present naturally which inspired the development of artificial ones like
Lead Zirconate Titanate (PZT) to be used as smart material to control the deformation. Mechanical and electrical coupling detailed in terms of the constitutive formulation bringing the stress, strain, material property, electric charge is given in the study [50]. The astonishing work [13] on shape, active control of FGM plate through piezoelectric material layers with numerical modeling become the benchmark for further studies and application. In this study, the closed-loop system working with displacement feedback is used. Shape control for laminated composite beams and plates coupled with piezoelectric layers is conducted with element-free Galerkin's method using first

S. A. Patare, swapnil925@iitb.ac.in | 'Civil Engineering Department, IIT Bombay, Powai, Mumbai 400076, India.

SN Applied Sciences (2021) 3:209| https://doi.org/10.1007/s42452-020-04121-y 
order shear deformation theory (FSDT), further from the finding of the study [22] it is suggested to locate actuator patches at high strain region for effective shape control. In the study [23] used a numerical solution with a radial basis function to perform static analysis and vibration control for a laminated composite plate using piezoelectric as a smart material. The numerical model developed in the study posses a better convergence rate than finite element method (FEM) based solution. Fiber-reinforced composites with piezo-layers are considered for static bending along with its control for FGM plate with a nonlinear variation of strains in study [32]. Simply supported at its edges two FGM layers are modeled with a homogeneous layer of a piezoelectric material sandwiched in between them to examine bending and free vibration analysis using statespace formulation in research conducted [7]. A Layer-wise approach in the finite element model for FGM plate coupled with piezoelectric layer is considered for static bending and dynamic analysis subjected to impulse loading, also the electric potential distribution is assumed layerwise giving sufficiently accurate results [42]. FGM plate resting on elastic foundation [2] is analyzed for a threedimensional exact solution with simply supported boundary condition which is integrated with piezoelectric layers using the Fourier series and state-space method. In the research effects of elastic foundation along with gradient index for electrical and mechanical loads is varied to study its effects. Geometric nonlinearity is taken into account while developing a mathematical model [3] for FGM with a numerical solution to get results for static bending under mechanical and electric loading. B-Spline finite strip method is used for static, vibration analysis of FGM plate coupled with skins of piezoelectric material in the study [25] demonstrating the advantage of B-Spline discretization over traditional FEM as it requires less electro-mechanical degrees of freedom. Reduction in stress concentration can be achieved through the smart application of piezoelectric material for host plate with a change in stiffness and thickness ratio is observed in the work done [11]. In the study [4] for composite beam coupled with piezoelectric patches genetic algorithm is used for optimizing shape control model based on Timoshenko's beam theory and numerical solution through FEM. To study the free vibration analysis of FGM plate with piezoelectric layers in terms of combination for a power law, aspect ratio [36] used four variable plate theory employing an analytical approach with Navier's solution for the simply supported case. Using FSDT based plate model [30] and cell-based smoothed discrete shear gap method while performing static shape and vibration control with closed-loop feedback algorithm based on displacement and velocity, further plate subjected to step, triangular, sinusoidal and explosive blast load is analyzed in the study.
To handle complex boundary conditions for the Levy type plate model in case of smart FGM plate [1] used spectral method while performing free vibration analysis. Taking forward the smart application of piezoelectric material [40] studied impact analysis on carbon nano-tubes (CNT) reinforced composite plate using Reddy's higher-order shear deformation theory [33], such that displacement is calculated with average acceleration Newmark's time integration method. Cracked FGM plate is studied for the uni and bi-directional critical buckling loads using the discrete shear gap method in context of numerical solution for the extended finite element analysis. It is observed in the study [24] that crack geometry can be independent of mesh grid, mesh distortion can be controlled, triangular elements can be easily generated even for complex geometry. Internal defects of FGM plate are mapped [57] through isogeometric analysis using FSDT for study of thermal buckling in the plate. To perform dynamic analysis with piezoelectric layers as fiber-reinforced composites coupled to cross-ply laminated composite [37] followed the analytical solution scheme with Navier's solution for the simply supported case of boundary conditions. In an interesting literature [49] study on the composite laminate microplate under the thermal environment is performed. The small scale effect is mapped properly through a combination of isogeometric analysis and modified couple stress theory under the bending and buckling effect. Reducing the stress concentrations, weight and/or improving the stiffness capacity is demonstrated through structural shape optimization using combination of extended isogeometric analysis and chaotic particle swarm optimization algorithm in the study [53] of structures with cutouts.

Plate theories following the equivalent single layer approach are well established in all domains of analysis like bending, buckling and vibration respectively. Classical work [18] was first to put forth a single layer model known as classical plate theory (CPT) gives accurate results only in the case of thin plates as the theory lacks incorporation of shear deformation effect. Later, second remarkable development [26] presented FSDT with the constant variation of shear strains through the thickness of plate which requires shear correction factor to get accurate results while performing complete analysis of thick plates. Higher order simple plate theory [33] considering the separated form of bending and shear deformation functions in the model is developed which is widely used. Results obtained from this theory were in good agreement with other higher order and three-dimensional plate theories as discussed in the study. The plate analysis model [33] was transformed into simple plate theory by splitting the transverse displacement into bending and shear with two separate functions for bending, shear each respectively minimizing the total number of governing variables to four was 
pioneering work [41]. Many researchers applied the basic logic of changing the shear deformation function by considering various mathematical forms satisfying the zero transverse shear stress condition, same models are listed and discussed in depth in this studies $[29,38]$. Polynomial based function was proposed with a cubic variation of shear deformation function along the thickness direction as seen $[15,31,35]$. Another polynomial based function was proposed [43] which gives good results to incorporate shear deformation effect in thick plates using four variable theory. Applying the capability of trigonometric sine function $[20,45,51]$ considered the shear deformation effect while developing the plate theory. Following the basic Taylor's series expansion based on plate displacement field's third term is assumed as hyperbolic shear deformation function was developed [44]. To map properly shear deformation, work done on plate analysis [16] proposed the use of an exponential function. All these models essentially follow the same logic of transverse shear stress to be zero at the top and bottom surface of the plate. Simple plate theory coupled with numerical method of isogeometric analysis through B-spline basis function such that $C^{1}$ continuity is satisfied [55]. Complex problem of laminated plate with cutout is handled in effective and simpler manner in the study [58] using simple FSDT along with numerical solution. New refined simple plate theory based on the kinematics of plate deformation through theory of elasticity is proposed for analysis of FGM plate for bending, buckling and vibration analysis in the study [52]. The new five variable shear deformation theory is developed in study [39] for sandwich beams and plates while examining various shape functions related to shear deformations. In this study flexural analysis is studied for simply supported FGM plate using closed-form Navier's solution. The small scale effect is mapped by proposing a new theory with three material scale parameters in the research [48]. In this study on the mechanical behavior of laminated composite plate is performed with free vibration analysis using Reddy's plate theory [33].

The solution of a partial differential system developed with the interaction of two directional variables ( $u, v$ along $x, y$ axes respectively) is complex especially when an analytical approach is used. The coupling of piezo layers to the core plate further elevates the complexity because of increased variables. To convert the partial differential system into ordinary applying the Fourier's series [28] obtained the solution for the plate with all four edges to be simply supported. To consider the more complex case of two opposite edges simply supported while remaining two to be in a combination of simple, fixed, or free support [21] devised the solution. [56] provided tables that consisted of beam functions along with roots of characteristic equations such that the solution for clamped, free and simply supported boundary conditions for uniform beams is obtained. In this study [17] an extended kantrovich method is used for simplifying the partial differential equation of the plate problem to be converted into an ordinary one. The iterative solution procedure ensures the convergence of the results and is independent of the starting solution. [12] proposed a superposition method to deal with all types of boundary conditions such that the obtained result proves the applicability of the proposed method. [6] solved the problem on natural frequencies of a rectangular plate using Rayleigh-Ritz developed an approximate analytical method with orthogonal polynomial for solving the problem under study. Hierarchical trigonometric [5] set of functions is developed considering the bending and rotation modes at both ends of beams. Using trigonometric functions as a solution to beam bending for complex boundary conditions terms considered in solution going as higher as up to 2048 was not affected due to computer round-off error while with the same problem solved by polynomial based functions failed at an order of 47.

In the present study simple analytical bending mathematical model is developed based on four variable polynomial based shear deformation plate theory for FGM plate coupled with piezoelectric layers followed by closed form solution obtained with the use of beam functions [8, $46,56]$. The second part of the study consists of the smart application of piezoelectric material to achieve the shape control of the FGM plate through a closed-loop system based on the displacement feedback algorithm [30]. Simple polynomial to be used as shear deformation function $[19,27,33,41]$ which is further compared with other mathematical models to prove its robustness, improves the approach towards simplicity of the current model. Shear deformation functions based models with mathematical form oriented from Polynomial, Trigonometric, Hyperbolic, and Exponential are presented here. The governing system is obtained with the aid of the Principle of virtual work for basic mechanical variables while Maxwell's condition is used to incorporate the part of the electrical variable. Flexural analysis of the FGM plate is presented as part of validation for the proposed model such that change in aspect ratio, material property, and boundary conditions are included. Smart application of piezoelectric material to control the static deflection of the FGM plate following the analytical approach in the form of a closed-loop system is developed with a demonstration in the form of numerical and graphical form. The novelty of the study is highlighted from the fact that in the field of plate analysis with a vast amount of literature available though every work appears similar, still the work presented here is unique in terms of the analytical approach to tackle the problem while most similar studies conducted it with a numerical approach. 


\section{Governing formulation}

\subsection{The displacement field}

Articulating displacement field [41] such that transverse displacement is assumed to be separated in two components as bending and shear each respectively. Though four variable are present in the displacement field, present theory effectively maps the shear deformation effect in case of increase in thickness of plate when compared with CPT [18], FSDT [26]. Figure 1 shows piezoelectric coupled FGM plate with coordinate axes assumed while going through derivation of mathematical model. Core FGM layer is of thickness $h$, while piezo-layers are made of thickness $h_{p}$. Size of plate is considered as $a * b$ along $x$ and $y$ axes respectively.

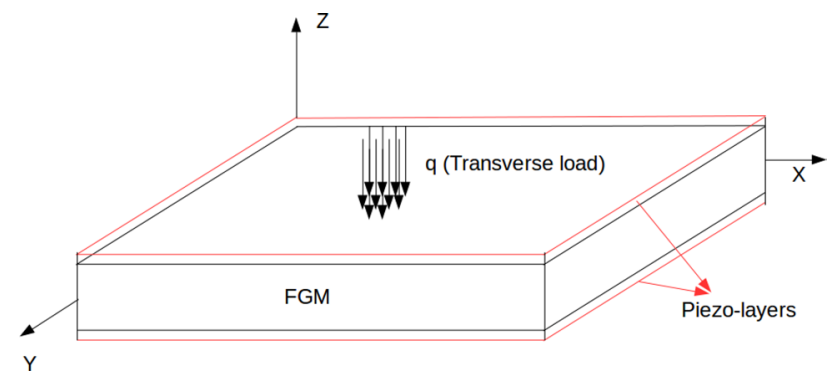

$u=u-z \frac{\partial w_{b}}{\partial x}-(F z) \frac{\partial w_{s}}{\partial x}$

$v=v-z \frac{\partial w_{b}}{\partial y}-(F z) \frac{\partial w_{s}}{\partial y}$

$w=w_{b}+w_{s}$

Plate theories have evolved based on the role of shear deformation function $[29,38]$ in case of increase in the plate thickness. Starting from simple polynomial going upto complex combination of polynomial, trigonometric, hyperbolic and exponential functions the plate theories are now matured in all sense. Six plate theories considered in the this study are as given in Table 1 for studying performance of each mathematical form in terms of shear deformation function, leading to approval of simple plate theory based on the sixth polynomial (PolyF3) function to be used shape control study of FGM plate. To satisfy the condition of transverse shear stress to be zero at top and bottom surface of plate, $G z=1-\frac{\partial F z}{\partial z}$ needs to be assumed.

\subsection{Linear strains}

Strains are given in Eq. (2) considering linear relation between strain-displacements for study of static bending and shape control of FGM plate through smart application of piezoelectric material.

Fig. 1 Piezoelectric coupled FGM plate model

Table 1 Plate theories based on shear deformation function in various mathematical forms

\begin{tabular}{llc}
\hline Plate theory & Shear deformation function & Model code \\
\hline Polynomial [15, 31, 35] & $\frac{-5 z}{4}+\frac{5 z^{3}}{3 h_{t}^{2}}$ & PolyF1 \\
Polynomial [43] & $\frac{-z}{4}+\frac{5 z^{3}}{3 h_{t}^{2}}$ & PolyF2 \\
Trigonometric [20, 45, 51] & $z-\left(\frac{h_{t}}{\pi} \sin \left(\frac{\pi z}{h_{t}}\right)\right)$ & TrigF \\
Hyperbolic [44] & $z-\left(z \cosh \left(\frac{1}{2}\right)-h_{t} \sinh \left(\frac{z}{h_{t}}\right)\right)$ & HyperF \\
Exponential [16] & $z-\left(z e^{\left.-2\left(\frac{z}{h_{t}}\right)^{2}\right)}\right.$ & ExpF \\
Polynomial [19, 27, 33, 41] & $\frac{4 z^{3}}{3 h_{t}^{2}}$ & PolyF3 \\
\hline
\end{tabular}




$$
\begin{aligned}
& \mathcal{E}_{x}=\frac{\partial u}{\partial x}-z \frac{\partial^{2} w_{b}}{\partial x^{2}}-(F z) \frac{\partial^{2} w_{s}}{\partial x^{2}} \\
& \mathcal{E}_{y}=\frac{\partial v}{\partial y}-z \frac{\partial^{2} w_{b}}{\partial y^{2}}-(F z) \frac{\partial^{2} w_{s}}{\partial y^{2}} \\
& \gamma_{x z}=\left(G_{z}\right) \frac{\partial w_{s}}{\partial x} \\
& \gamma_{y z}=\left(G_{z}\right) \frac{\partial w_{s}}{\partial y} \\
& \gamma_{x y}=\frac{\partial u}{\partial y}+\frac{\partial v}{\partial x}-2 z \frac{\partial^{2} w_{b}}{\partial x \partial y}-2 z \frac{\partial^{2} w_{s}}{\partial x \partial y}
\end{aligned}
$$

\subsection{Complementary stresses}

The complementary stress-strain relations [50] for piezoelectric layers coupled to FGM plate are as given in Eq. (3).

$$
\begin{aligned}
& \sigma_{x}=\bar{Q}_{11} \mathcal{E}_{x}+\bar{Q}_{12} \mathcal{E}_{y}-e_{31} E_{z} \\
& \sigma_{y}=\bar{Q}_{21} \mathcal{E}_{x}+\bar{Q}_{22} \mathcal{E}_{y}-e_{32} E_{z} \\
& \sigma_{x z}=\bar{Q}_{44} \gamma_{x z}-e_{15} E_{x} \\
& \sigma_{y z}=\bar{Q}_{55} \gamma_{y z}-e_{24} E_{y} \\
& \sigma_{x y}=\bar{Q}_{66} \gamma_{x y}
\end{aligned}
$$

In above relations, $\bar{Q}=Q$ is the constant assumed for FGM core plate while $\bar{Q}=C$ for material of piezoelectric layers. The material property variation is mapped by power law $[14,34]$ through the thickness direction of the FGM plate. $e_{15}, e_{24}, e_{31}, e_{32}, e_{33}$ are piezoelectric constants and electric field intensity is given by $E_{x}, E_{y}$ and $E_{z}$ respectively. The material property elastic constants are given below along with the power law for through thickness material variation of FGM plate, assuming behavior of an isotropic material.

$$
\begin{aligned}
& \bar{Q}_{11}=\bar{Q}_{22}=\frac{E(z)}{\left(1-\mu_{z}^{2}\right)^{\prime}} \\
& \bar{Q}_{12}=\bar{Q}_{21}=\frac{\mu_{z} E(z)}{\left(1-\mu_{z}^{2}\right)^{\prime}} \\
& \bar{Q}_{44}=\bar{Q}_{55}=\bar{Q}_{66}=\frac{E(z)}{2\left[1+\mu_{z}\right]} \\
& E(z)=E(m)+[E(c)-E(m)]\left(\frac{1}{2}+\frac{z}{h}\right)^{N}
\end{aligned}
$$

\subsection{Principle of virtual work}

To obtain the governing equations for piezoelectric coupled FGM plate principle of virtual work is used. Work done by transverse load $q$ distributed over the surface of plate results in change of stress-strain over the volume domain of plate is considered [Eq. (4)].

$$
\begin{gathered}
\int_{V}\left[\sigma_{x} \delta \mathcal{E}_{x}+\sigma_{y} \delta \mathcal{E}_{y}+\sigma_{x y} \delta \gamma_{x y}+\sigma_{x z} \delta \gamma_{x z}+\sigma_{y z} \delta \gamma_{y z}\right] d V \\
-\int_{0}^{a} \int_{0}^{b} q \delta\left(w_{b}+w_{s}\right) d A=0
\end{gathered}
$$

Simplifying Eq. (4) by taking out the thickness integrals of stress resultants given in Eq. (6) each with $h_{T}=(h / 2)+h_{p}$ and $h_{B}=-(h / 2)-h_{p}$ taken as the limits.

$$
\begin{gathered}
\int_{0}^{a} \int_{0}^{b}\left\{N_{x} \frac{\partial \delta u}{\partial x}+N_{y} \frac{\partial \delta v}{\partial y}+N_{x y}\left[\frac{\partial \delta u}{\partial y}+\frac{\partial \delta v}{\partial x}\right]\right. \\
-M_{x}^{b} \frac{\partial^{2} \delta w_{b}}{\partial x^{2}}-M_{y}^{b} \frac{\partial^{2} \delta w_{b}}{\partial y^{2}}-2 M_{x y}^{b} \frac{\partial^{2} \delta w_{b}}{\partial x \partial y} \\
-M_{x}^{s} \frac{\partial^{2} \delta w_{s}}{\partial x^{2}}-M_{y}^{s} \frac{\partial^{2} \delta w_{s}}{\partial y^{2}}-2 M_{x y}^{s} \frac{\partial^{2} \delta w_{s}}{\partial x \partial y} \\
\left.+Q_{x z} \frac{\partial \delta w_{s}}{\partial x}+Q_{y z} \frac{\partial \delta w_{s}}{\partial y}\right\} d x d y \\
-\int_{0}^{a} \int_{0}^{b} q \delta\left(w_{b}+w_{s}\right) d x d y=0 \\
\left(N_{x^{\prime}}, N_{y}, N_{x y}\right)=\int_{h_{B}}^{h_{T}}\left(\sigma_{x}, \sigma_{y}, \sigma_{x y}\right) d z \\
\left(M_{x^{\prime}}^{b}, M_{y}^{b}, M_{x y}^{b}\right)=\int_{h_{B}}^{h_{T}}\left(\sigma_{x}, \sigma_{y}, \sigma_{x y}\right) z d z \\
\left(M_{x^{\prime}}^{s}, M_{y^{\prime}}^{s}, M_{x y}^{s}\right)=\int_{h_{B}}^{h_{T}}\left(\sigma_{x}, \sigma_{y}, \sigma_{x y}\right)(F z) d z \\
\left(Q_{x z}, Q_{y z}\right)=\int_{h_{B}}^{h_{T}}\left(\sigma_{x z}, \sigma_{y z}\right)(G z) d z
\end{gathered}
$$

\section{Governing equations}

Collecting the coefficients of four virtual displacements $\left(\delta u, \delta v, \delta w_{b}\right.$ and $\left.\delta w_{s}\right)$ together as demonstrated in Eq. (7), is obtained by performing integration by parts for Eq. (5). The associated boundary conditions are presented in Table 2 valid along the plate edges coming out along with the governing equation. 
Table 2 Associated boundary conditions

\begin{tabular}{lllll}
\hline At edge $\mathrm{x}=0, \mathrm{a}$ & & & At edge $\mathrm{y}=0, \mathrm{~b}$ & \\
\cline { 1 - 2 } \cline { 4 - 5 } Geometric & Natural & & Geometric & Natural \\
\hline$u$ & $N_{x}=0$ & & $N_{y}=0$ \\
$v$ & $N_{x y}=0$ & & $w_{b}$ & $N_{x y}=0$ \\
$w_{b}$ & $\frac{\partial M_{x}^{b}}{\partial x}+\frac{\partial M_{x y}^{b}}{\partial y}=0$ & & $\frac{\partial M_{y}^{b}}{\partial y}+\frac{\partial M_{x y}^{b}}{\partial x}=0$ \\
$w_{s}$ & $\frac{\partial M_{x}^{s}}{\partial x}+\frac{\partial M_{x y}^{s}}{\partial y}-Q_{x z}=0$ & $w_{s}$ & $\frac{\partial M_{y}^{s}}{\partial y}+\frac{\partial M_{x y}^{s}}{\partial x}-Q_{y z}=0$ \\
$\frac{\partial w_{b}}{\partial x}$ & $M_{x}^{b}=0$ & $\frac{\partial w_{b}}{\partial y}$ & $M_{y}^{b}=0$ \\
$\frac{\partial w_{b}}{\partial y}$ & $M_{x y}^{b}=0$ & $\frac{\partial w_{b}}{\partial x}$ & $M_{x y}^{b}=0$ \\
$\frac{\partial w_{s}}{\partial x}$ & $M_{x}^{s}=0$ & $\frac{\partial w_{s}}{\partial y}$ & $M_{y}^{s}=0$ \\
$\frac{\partial w_{s}}{\partial y}$ & $M_{x y}^{s}=0$ & $\frac{\partial w_{s}}{\partial x}$ & $M_{x y}^{s}=0$ \\
\hline
\end{tabular}

$$
\begin{aligned}
\delta u & : \frac{\partial N_{x}}{\partial x}+\frac{\partial N_{x y}}{\partial y}=0 \\
\delta v & : \frac{\partial N_{y}}{\partial y}+\frac{\partial N_{x y}}{\partial x}=0 \\
\delta w_{b}: & \frac{\partial^{2} M_{x}^{b}}{\partial x^{2}}+2 \frac{\partial^{2} M_{x y}^{b}}{\partial x \partial y}+\frac{\partial^{2} M_{y}^{b}}{\partial y^{2}}+q_{w}=0 \\
\delta w_{s}: & \frac{\partial^{2} M_{x}^{s}}{\partial x^{2}}+2 \frac{\partial^{2} M_{x y}^{s}}{\partial x \partial y}+\frac{\partial^{2} M_{y}^{s}}{\partial y^{2}} \\
& +\frac{\partial Q_{x z}}{\partial x}+\frac{\partial Q_{y z}}{\partial y}+q_{w}=0
\end{aligned}
$$

In above equation term $q_{w}$ is the simplified form of work done by transverse loading $q$, $\left(-\int_{0}^{a} \int_{o}^{b} q\left(\delta w_{b}+\delta w_{s}\right) d x d y\right)$

Final form of governing Eq. (8) is obtained by substituting for stresses in terms of strains in Eq. (6) for stress resultants. Later these expanded stress resultants are substituted in Eq. (7).

$$
\begin{aligned}
& A_{11} \frac{\partial^{2} u}{\partial x^{2}}+A_{66} \frac{\partial^{2} u}{\partial y^{2}}+\left(A_{12}+A_{66}\right) \frac{\partial^{2} v}{\partial x \partial y}-B_{11} \frac{\partial^{3} w_{b}}{\partial x^{3}} \\
& -\left(B_{12}+2 B_{66}\right) \frac{\partial^{3} w_{b}}{\partial x \partial y^{2}}-B_{11}^{s} \frac{\partial^{3} w_{s}}{\partial x^{3}}-\left(B_{12}^{s}+2 B_{66}^{s}\right) \\
& \frac{\partial^{3} w_{s}}{\partial x \partial y^{2}}+\mu_{1} \frac{\partial \Pi_{T}}{\partial x}+\mu_{2} \frac{\partial \Pi_{B}}{\partial x}=0
\end{aligned}
$$

$$
\begin{aligned}
& \left(A_{12}+A_{66}\right) \frac{\partial^{2} u}{\partial x \partial y}+A_{66} \frac{\partial^{2} v}{\partial x^{2}}+A_{22} \frac{\partial^{2} v}{\partial y^{2}}-\left(B_{12}+2 B_{66}\right) \\
& \frac{\partial^{3} w_{b}}{\partial x^{2} \partial y}-B_{22} \frac{\partial^{3} w_{b}}{\partial y^{3}}-\left(B_{12}^{s}+2 B_{66}^{s}\right) \frac{\partial^{3} w_{s}}{\partial x^{2} \partial y}-B_{22}^{s} \frac{\partial^{3} w_{s}}{\partial y^{3}} \\
& +\mu_{3} \frac{\partial \Pi_{T}}{\partial y}+\mu_{4} \frac{\partial \Pi_{B}}{\partial y}=0 \\
& B_{11} \frac{\partial^{3} u}{\partial x^{3}}+\left(B_{12}+2 B_{66}\right) \frac{\partial^{3} u}{\partial x \partial y^{2}}+\left(B_{12}+2 B_{66}\right) \frac{\partial^{3} v}{\partial x^{2} \partial y} \\
& +B_{22} \frac{\partial^{3} v}{\partial y^{3}}-D_{11} \frac{\partial^{4} w_{b}}{\partial x^{4}}-2\left(D_{12}+2 D_{66}\right) \frac{\partial^{4} w_{b}}{\partial x^{2} \partial y^{2}} \\
& \quad-D_{22} \frac{\partial^{4} w_{b}}{\partial y^{4}}-D_{11}^{s} \frac{\partial^{4} w_{s}}{\partial x^{4}}-2\left(D_{12}^{s}+2 D_{66}^{s}\right) \frac{\partial^{4} w_{s}}{\partial x^{2} \partial y^{2}} \\
& \quad-D_{22}^{s} \frac{\partial^{4} w_{s}}{\partial y^{4}}+\mu_{5} \frac{\partial^{2} \Pi_{T}}{\partial x^{2}}+\mu_{7} \frac{\partial^{2} \Pi_{T}}{\partial y^{2}}+\mu_{6} \frac{\partial^{2} \Pi_{B}}{\partial x^{2}} \\
& \quad+\mu_{8} \frac{\partial^{2} \Pi_{B}}{\partial y^{2}}+q_{w}=0
\end{aligned}
$$

$$
\begin{aligned}
B_{11}^{s} & \frac{\partial^{3} u}{\partial x^{3}}+\left(B_{12}^{s}+2 B_{66}^{s}\right) \frac{\partial^{3} u}{\partial x \partial y^{2}}+\left(B_{12}^{s}+2 B_{66}^{s}\right) \frac{\partial^{3} v}{\partial x^{2} \partial y} \\
+ & B_{22}^{s} \frac{\partial^{3} v}{\partial y^{3}}-D_{11}^{s} \frac{\partial^{4} w_{b}}{\partial x^{4}}-2\left(D_{12}^{s}+2 D_{66}^{s}\right) \frac{\partial^{4} w_{b}}{\partial x^{2} \partial y^{2}} \\
& -D_{22}^{s} \frac{\partial^{4} w_{b}}{\partial y^{4}}-H_{11}^{s} \frac{\partial^{4} w_{s}}{\partial x^{4}}-2\left(H_{12}^{s}+2 H_{66}^{s}\right) \frac{\partial^{4} w_{s}}{\partial x^{2} \partial y^{2}} \\
& -H_{22}^{s} \frac{\partial^{4} w_{s}}{\partial y^{4}}+A_{44} \frac{\partial^{2} w_{s}}{\partial x^{2}}+A_{55} \frac{\partial^{2} w_{s}}{\partial y^{2}}
\end{aligned}
$$




$$
\begin{aligned}
& +\left(\mu_{9}+\mu_{15}\right) \frac{\partial^{2} \Pi_{T}}{\partial x^{2}}+\left(\mu_{11}+\mu_{17}\right) \frac{\partial^{2} \Pi_{T}}{\partial y^{2}} \\
& +\left(\mu_{10}+\mu_{16}\right) \frac{\partial^{2} \Pi_{B}}{\partial x^{2}}+\left(\mu_{12}+\mu_{18}\right) \frac{\partial^{2} \Pi_{B}}{\partial y^{2}}+q_{w}=0
\end{aligned}
$$

Elastic material property in the form of thickness integral are taken as;

$$
\begin{aligned}
&\left(A_{11}, A_{12}, A_{22}, A_{66}\right) \\
&= \int_{-h / 2}^{h / 2}\left(Q_{11}, Q_{12}, Q_{22}, Q_{66}\right) d z \\
&+2 \int_{h / 2}^{h_{T}}\left(C_{11}, C_{12}, C_{22}, C_{66}\right) d z \\
& {[3 p t]\left(B_{11}, B_{12}, B_{22}, B_{66}\right) } \\
&=\int_{-h / 2}^{h / 2}\left(Q_{11}, Q_{12}, Q_{22}, Q_{66}\right) z d z \\
&+2 \int_{h / 2}^{h_{T}}\left(C_{11}, C_{12}, C_{22}, C_{66}\right) z d z \\
& {[3 p t]\left(B_{11}^{s}, B_{12}^{s}, B_{22}^{s}, B_{66}^{s}\right) } \\
&=\int_{-h / 2}^{h / 2}\left(Q_{11}, Q_{12}, Q_{22}, Q_{66}\right)(F z) d z \\
&+2 \int_{h / 2}^{h_{T}}\left(C_{11}, C_{12}, C_{22}, C_{66}\right)(F z) d z \\
& {[3 p t]\left(A_{44}, A_{55}\right)=} \int_{-h / 2}^{h / 2}\left(Q_{44}, Q_{55}\right)(G z)^{2} d z \\
&+2 \int_{h / 2}^{h_{T}}\left(C_{44}, C_{55}\right)(G z)^{2} d z \\
& {[3 p t]\left(H_{11}^{s}, H_{12}^{s}, H_{22}^{s}, H_{66}^{s}\right) } \int_{-h / 2}^{h / 2}\left(Q_{11}, Q_{12}, Q_{22}, Q_{66}\right)(F z)^{2} d z \\
&+2 \int_{h / 2}^{h_{T}}\left(C_{11}, C_{12}, C_{22}, C_{66}\right)(F z)^{2} d z \\
&= \int_{-h / 2}^{h / 2}\left(Q_{11}, Q_{12}, Q_{22}, Q_{66}\right) z^{2} d z \\
&+2 \int_{h / 2}^{h_{T}}\left(C_{11}, C_{12}, C_{22}, C_{66}\right) z^{2} d z \\
& {[3 p t]\left(D_{11}^{s}, D_{12}^{s}, D_{22}^{s}, D_{66}^{s}\right) } \\
&= \int_{-h / 2}^{h / 2}\left(Q_{11}, Q_{12}, Q_{22}, Q_{66}\right) z(F z) d z \\
&+2 \int_{11}^{h_{T}}\left(C_{11}, C_{12}, C_{22}, C_{66}\right) z(F z) d z \\
&\left.D_{22}, D_{66}\right) \\
& \\
&
\end{aligned}
$$

Electric properties along the thickness direction taken out as integrals to simply the formulation are given as below;

$$
\begin{aligned}
\left(\mu_{1}, \mu_{3}\right) & =\int_{h / 2}^{h_{T}}\left(e_{31}, e_{32}\right) \frac{\partial \Phi_{T}}{\partial z} d z \\
\left(\mu_{2}, \mu_{4}\right) & =\int_{h_{B}}^{-h / 2}\left(e_{31}, e_{32}\right) \frac{\partial \Phi_{B}}{\partial z} d z \\
\left(\mu_{5}, \mu_{7}\right) & =\int_{h / 2}^{h_{T}}\left(e_{31}, e_{32}\right) \frac{\partial \Phi_{T}}{\partial z} z d z \\
\left(\mu_{6}, \mu_{8}\right) & =\int_{h_{B}}^{-h / 2}\left(e_{31}, e_{32}\right) \frac{\partial \Phi_{B}}{\partial z} z d z \\
\left(\mu_{9}, \mu_{11}\right) & =\int_{h / 2}^{h_{T}}\left(e_{31}, e_{32}\right) \frac{\partial \Phi_{T}}{\partial z}(F z) d z \\
\left(\mu_{10}, \mu_{12}\right) & =\int_{h_{B}}^{-h / 2}\left(e_{31}, e_{32}\right) \frac{\partial \Phi_{B}}{\partial z}(F z) d z \\
\left(\mu_{15}, \mu_{17}\right) & =\int_{h / 2}^{h_{T}}\left(e_{15}, e_{24}\right)(G z) \Phi_{T} d z \\
\left(\mu_{16}, \mu_{18}\right) & =\int_{h_{B}}^{-h / 2}\left(e_{15}, e_{24}\right)(G z) \Phi_{B} d z
\end{aligned}
$$

\section{Piezoelectric coupling}

\subsection{Electric potential function}

Electrical potential function varying along the thickness of piezoelectric layers is second order polynomial verified in the study [54] with finite element which is used in present study. Same function was also used for free vibration analysis by [10] with FSDT and [36] with four variable refined plate theory to study free vibration analysis for FGM plate.

$$
\begin{gathered}
\Phi_{T}=\Pi_{T}\left[1-\left(\frac{z-h-h_{p} / 2}{h_{p} / 2}\right)^{2}\right] \\
\left\langle h / 2 \leq z \leq(h / 2)+h_{p}\right\rangle \\
\Phi_{B}=\Pi_{B}\left[1-\left(\frac{-z-h-h_{p} / 2}{h_{p} / 2}\right)^{2}\right] \\
\left\langle-(h / 2)-h_{p} \leq z \leq-h / 2\right\rangle
\end{gathered}
$$

\subsection{Electric displacement}

Mechanical equilibrium is satisfied as per the detailed formulation given in section (3) with the four governing equations [Eq. (7)], similarly Maxwell's electrical condition needs to be satisfied given in Eq. (14). The electric 
displacement is as detailed in Eq. (13) in terms of strain and electric field intensities.

$D_{x}=e_{15} \gamma_{x z}+\Omega_{11} E_{x}$

$D_{y}=e_{24} \gamma_{y z}+\Omega_{22} E_{y}$

$D_{z}=e_{31} \mathcal{E}_{x}+e_{32} \mathcal{E}_{y}+\Omega_{33} E_{z}$

\subsection{Fifth governing equation}

Piezoelectric coupling increases number of variables to five hence additional equation is obtained by satisfying Maxwell's electrical condition. Simplified form of this equation is given in Eq. (15), after substituting for electric displacement in Eq. (14).

$$
\begin{aligned}
& \int_{h / 2}^{h_{T}}\left(\frac{\partial D_{x}}{\partial x}+\frac{\partial D_{y}}{\partial y}+\frac{\partial D_{z}}{\partial z}\right) d z \\
& \quad+\int_{h_{B}}^{-h / 2}\left(\frac{\partial D_{x}}{\partial x}+\frac{\partial D_{y}}{\partial y}+\frac{\partial D_{z}}{\partial z}\right) d z=0 \\
& -\lambda_{5} \frac{\partial^{2} w_{b}}{\partial x^{2}}-\lambda_{6} \frac{\partial^{2} w_{b}}{\partial y^{2}}+\lambda_{1} \frac{\partial^{2} w_{s}}{\partial x^{2}}+\lambda_{2} \frac{\partial^{2} w_{s}}{\partial y^{2}}-\lambda_{7} \frac{\partial^{2} \Pi_{T}}{\partial x^{2}} \\
& -\lambda_{9} \frac{\partial^{2} \Pi_{T}}{\partial y^{2}}-\lambda_{8} \frac{\partial^{2} \Pi_{B}}{\partial x^{2}}-\lambda_{10} \frac{\partial^{2} \Pi_{B}}{\partial y^{2}} \\
& -\lambda_{12} \Pi_{T}-\lambda_{13} \Pi_{B}=0
\end{aligned}
$$

The constants for thickness integrals based on the properties of piezoelectric layers are given below;

$$
\begin{aligned}
\lambda_{1}= & \int_{h / 2}^{h_{T}}\left(e_{15}(G z)-e_{31} \frac{\partial F z}{\partial z}\right) d z \\
& +\int_{h_{B}}^{-h / 2}\left(e_{15}(G z)-e_{31} \frac{\partial F z}{\partial z}\right) d z \\
\lambda_{2}= & \int_{h / 2}^{h_{T}}\left(e_{24}(G z)-e_{32} \frac{\partial F z}{\partial z}\right) d z \\
& +\int_{h_{B}}^{-h / 2}\left(e_{24}(G z)-e_{32} \frac{\partial F z}{\partial z}\right) d z \\
\lambda_{5}= & \int_{h / 2}^{h_{T}} e_{31} d z+\int_{h_{B}}^{-h / 2} e_{31} d z \\
\lambda_{6}= & \int_{h / 2}^{h_{T}} e_{32} d z+\int_{h_{B}}^{-h / 2} e_{32} d z \\
\lambda_{7}= & \int_{h / 2}^{h_{T}}\left(\Omega_{11} \Phi_{T}\right) d z, \lambda_{8}=\int_{h_{B}}^{-h / 2}\left(\Omega_{11} \Phi_{B}\right) d z \\
\lambda_{9}= & \int_{h / 2}^{h_{T}}\left(\Omega_{22} \Phi_{T}\right) d z, \lambda_{10}=\int_{h_{B}}^{-h / 2}\left(\Omega_{22} \Phi_{B}\right) d z \\
\lambda_{12}= & \int_{h / 2}^{h_{T}} \Omega_{33} \frac{\partial^{2} \Phi_{T}}{\partial z^{2}} d z \\
\lambda_{13}= & \int_{h_{B}}^{-h / 2} \Omega_{33} \frac{\partial^{2} \Phi_{B}}{\partial z^{2}} d z
\end{aligned}
$$

\subsection{Active feedback system}

The constant displacement feedback control algorithm [30] is used for shape control of FGM plate assuming bottom piezoelectric layer acting as sensor while top acting as actuator given as $\Pi_{T}=G_{d} * \Pi_{B}$. To convert the system in single input single output, the electric variable in bottom layer is assumed as $\Pi_{B}=\Pi$.

\section{Analytical solution}

Five coupled equation forms complex system for bending analysis of FGM plate due to piezoelectric layers. Solution to be assumed for this highly coupled partial differential governing system is obtained for five basic variables based on the beam functions $[8,46,56]$ considering the combination of simply supported and clamped boundary conditions. The expansion for variables is given in Eq. (17) based on satisfaction of geometric boundary conditions. 


$$
\begin{aligned}
& u=\sum_{m=1}^{\infty} \sum_{n=1}^{\infty} U_{m n} \frac{\partial X_{m}}{\partial x} Y_{n}, \\
& v=\sum_{m=1}^{\infty} \sum_{n=1}^{\infty} v_{m n} X_{m} \frac{\partial Y_{n}}{\partial y}, \\
& w_{b}=\sum_{m=1}^{\infty} \sum_{n=1}^{\infty} w_{m n}^{b} X_{m} Y_{n^{\prime}} \\
& w_{s}=\sum_{m=1}^{\infty} \sum_{n=1}^{\infty} W_{m n}^{s} X_{m} Y_{n^{\prime}} \\
& \Pi=\sum_{m=1}^{\infty} \sum_{n=1}^{\infty} \Pi_{m n} X_{m} Y_{n}
\end{aligned}
$$

Simply-supported (SS):

$$
\begin{aligned}
v & =w_{b}=w_{s}=N_{x}=M_{x}^{b}=M_{x}^{s}=0 \text { at } x=0, a \\
{[3 p t] u } & =w_{b}=w_{s}=N_{y}=M_{y}^{b}=M_{y}^{s}=0 \text { at } y=0, b
\end{aligned}
$$

\section{Clamped (CC):}

$$
\begin{array}{r}
u=v=w_{b}=\frac{\partial w_{b}}{\partial x}=\frac{\partial w_{b}}{\partial y}=w_{s}=\frac{\partial w_{s}}{\partial x}=\frac{\partial w_{s}}{\partial y}=0 \\
\text { at } x=0, \text { a and at } y=0, b
\end{array}
$$

Table 3 consists beam shape functions which are obtained as a solution to frequency equation corresponding to beams with respective boundaries. The symbols used in Table 3 are interpreted as CC is clamped-clamped opposite edges, CS is clamped-simply supported while SS is for both opposite edges simply supported. To simplify the lengthy mathematical equations following coding is used such

\begin{tabular}{|c|c|c|c|c|}
\hline \multirow[t]{2}{*}{ Boundary type } & \multicolumn{2}{|l|}{ Boundary condition } & \multirow{2}{*}{$\begin{array}{l}\text { Eigenfunction } \\
X_{m}(x), Y_{n}(y)\end{array}$} & \multirow{2}{*}{$\begin{array}{l}\text { Roots of frequency equation } \\
\lambda_{m}, \lambda_{n}\end{array}$} \\
\hline & $x=0, a$ & $y=0, b$ & & \\
\hline \multirow[t]{4}{*}{ CC } & $X_{m}(0)=X_{m}^{\prime}(0)=0$ & $Y_{n}(0)=Y_{n}^{\prime}(0)=0$ & $J\left(\frac{\lambda_{m} x}{a}\right)-\frac{J\left(\lambda_{m}\right)}{H\left(\lambda_{m}\right)} H\left(\frac{\lambda_{m} x}{a}\right)$ & $\lambda_{1}=4.7300, \lambda_{2}=7.8532$ \\
\hline & $X_{m}(a)=X_{m}^{\prime}(a)=0$ & $Y_{n}(b)=Y_{n}^{\prime}(b)=0$ & $J\left(\frac{\lambda_{n} y}{b}\right)-\frac{J\left(\lambda_{n}\right)}{H\left(\lambda_{n}\right)} H\left(\frac{\lambda_{n} y}{b}\right)$ & $\lambda_{3}=10.9956, \lambda_{4}=14.1372$ \\
\hline & & & & For large $m, \lambda_{m} \approx \frac{(2 m+1) \pi}{2}$ \\
\hline & & & & Forlarae m $\lambda_{n} \approx \frac{(2 n+1) \pi}{2}$ \\
\hline \multirow[t]{4}{*}{ CS } & $X_{m}(0)=X_{m}^{\prime}(0)=0$ & $Y_{n}(0)=Y_{n}^{\prime}(0)=0$ & $J\left(\frac{\lambda_{m} x}{a}\right)-\frac{J\left(\lambda_{m}\right)}{H\left(\lambda_{m}\right)} H\left(\frac{\lambda_{m} x}{a}\right)$ & $\lambda_{1}=3.9266, \lambda_{2}=7.0686$ \\
\hline & $X_{m}(a)=X_{m}^{\prime \prime}(a)=0$ & $Y_{n}(b)=Y_{n}^{\prime \prime}(b)=0$ & $J\left(\frac{\lambda_{n} y}{b}\right)-\frac{J\left(\lambda_{n}\right)}{H\left(\lambda_{n}\right)} H\left(\frac{\lambda_{n} y}{b}\right)$ & $\lambda_{3}=10.2102, \lambda_{4}=13.3518$ \\
\hline & & & & For large $m, \lambda_{m} \approx \frac{(4 m+1) \pi}{4}$ \\
\hline & & & & For larqe $\mathrm{m} \lambda_{n} \approx \frac{(4 n+1) \pi}{4}$ \\
\hline \multirow[t]{2}{*}{ SS } & $X_{m}(0)=X_{m}^{\prime \prime}(0)=0$ & $Y_{n}(0)=Y_{n}^{\prime \prime}(0)=0$ & $\sin \left(\frac{m \pi x}{a}\right)$ & $\lambda_{m}=m \pi$ \\
\hline & $X_{m}(a)=X_{m}^{\prime \prime}(a)=0$ & $Y_{n}(b)=Y_{n}^{\prime \prime}(b)=0$ & $\sin \left(\frac{n \pi y}{b}\right)$ & $\lambda_{n}=n \pi$ \\
\hline
\end{tabular}
that $\lambda_{m}, \lambda_{n}$ are the roots of frequency equation.

$$
\begin{aligned}
& H\left(\frac{\lambda_{m} x}{a}\right)=\sinh \left(\frac{\lambda_{m} x}{a}\right)-\sin \left(\frac{\lambda_{m} x}{a}\right), \\
& H\left(\frac{\lambda_{n} y}{b}\right)=\sinh \left(\frac{\lambda_{n} y}{b}\right)-\sin \left(\frac{\lambda_{n} y}{b}\right), \\
& J\left(\frac{\lambda_{m} x}{a}\right)=\cosh \left(\frac{\lambda_{m} x}{a}\right)-\cos \left(\frac{\lambda_{m} x}{a}\right), \\
& J\left(\frac{\lambda_{n} y}{b}\right)=\cosh \left(\frac{\lambda_{n} y}{b}\right)-\cos \left(\frac{\lambda_{n} y}{b}\right)
\end{aligned}
$$

The computer code adoptable stiffness matrix terms which can be used in any suitable programming tool obtained from the $5 * 5$ order piezoelectric coupled FGM plate governing system when solution from Eq. (17) is substituted in Eqs. (8 and 15).

Table 3 Beam shape functions. 


$$
\begin{aligned}
K_{11}= & A_{11} Y_{12}+A_{66} Y_{8} \\
K_{12}= & \left(A_{12}+A_{66}\right) Y_{8} \\
K_{13}= & -B_{11} Y_{12}-\left(B_{12}+2 B_{66}\right) Y_{8} \\
K_{14}= & -B_{11}^{s} Y_{12}-\left(B_{12}^{s}+2 B_{66}^{s}\right) Y_{8} \\
K_{15}= & \left(\mu_{1}+\mu_{2}\right) Y_{6} \\
{[6 p t] K_{21}=} & \left(A_{12}+A_{66}\right) Y_{10} \\
K_{22}= & A_{22} Y_{4}+A_{66} Y_{10} \\
K_{23}= & -B_{22} Y_{4}-\left(B_{12}+2 B_{66}\right) Y_{10} \\
K_{24}= & -B_{22}^{s} Y_{4}-\left(B_{12}^{s}+2 B_{66}^{s}\right) Y_{10} \\
K_{25}= & \left(\mu_{3}+\mu_{4}\right) Y_{2} \\
{[6 p t] K_{31}=} & B_{11} Y_{13}+\left(B_{12}+2 B_{66}\right) Y_{11} \\
K_{32}= & \left(B_{12}+2 B_{66}\right) Y_{11}+B_{22} Y_{5} \\
K_{33}= & -D_{11} Y_{13}-2\left(D_{12}+2 D_{66}\right) Y_{11} \\
& -D_{22} Y_{5} \\
K_{34}= & -D_{11}^{s} Y_{13}-2\left(D_{12}^{s}+2 D_{66}^{s}\right) Y_{11} \\
& -D_{22}^{s} Y_{5} \\
K_{35}= & \left(\mu_{5}+\mu_{6}\right) Y_{9}+\left(\mu_{7}+\mu_{8}\right) Y_{3} ; \\
{[6 p t] K_{41}=} & B_{11}^{s} Y_{13}+\left(B_{12}^{s}+2 B_{66}^{s}\right) Y_{11} \\
K_{42}= & \left(B_{12}^{s}+2 B_{66}^{s}\right) Y_{11}+B_{22}^{s} Y_{5} \\
K_{43}= & -D_{11}^{s} Y_{13}-2\left(D_{12}^{s}+2 D_{66}^{s}\right) Y_{11} \\
& -D_{22}^{s} Y_{5} \\
K_{44}= & \left.\left.-H_{11}^{s} Y_{13}-2\left(H_{12}^{s}+2 H_{66}^{s}\right) Y_{11}+\lambda_{13}\right) Y_{1}\right) \\
& -H_{22}^{s} Y_{5}-A_{44}^{s} Y_{9}-A_{55}^{s} Y_{3} \\
K_{45}= & \left(\mu_{9}+\mu_{15}+\mu_{10}+\mu_{16}\right) Y_{9} \\
& +\left(\mu_{11}+\mu_{17}+\mu_{12}+\mu_{18}\right) Y_{3} \\
K_{51}= & 0 \\
K_{52}= & 0 \\
K_{53}= & -\lambda_{5} Y_{9}-\lambda_{6} Y_{3 \prime} \\
K_{54}= & \lambda_{1} Y_{9}+\lambda_{2} Y_{3 \prime} \\
K_{55}= & -\left(\left(\lambda_{7}+\lambda_{8}\right) Y_{9}+\left(\lambda_{9}+\lambda_{10}\right) Y_{3}\right. \\
& +\left(\lambda_{12}\right. \\
&
\end{aligned}
$$

Further simplified form of integrals used in stiffness terms valid over the area domain of plate are considered separately as given below to make formulation simple;

$$
\begin{aligned}
\left(Y_{6}, Y_{8}, Y_{12}\right)= & \int_{0}^{a} \int_{0}^{b}\left(X_{m}^{\prime} Y_{n^{\prime}} X_{m}^{\prime} Y_{n^{\prime}}^{\prime \prime}\right. \\
& \left.X_{m}^{\prime \prime \prime} Y_{n}\right) X_{m}^{\prime} Y_{n} d x d y, \\
\left(Y_{2}, Y_{4}, Y_{10}\right)= & \int_{0}^{a} \int_{0}^{b}\left(X_{m} Y_{n^{\prime}}^{\prime} X_{m} Y_{n^{\prime \prime}}^{\prime \prime \prime}\right. \\
& \left.X_{m}^{\prime \prime} Y_{n}^{\prime}\right) X_{m} Y_{n}^{\prime} d x d y, \\
\left(Y_{1}, Y_{3}, Y_{5}\right)= & \int_{0}^{a} \int_{0}^{b}\left(X_{m} Y_{n^{\prime}} X_{m} Y_{n^{\prime}}^{\prime \prime}\right. \\
& \left.X_{m} Y_{n}^{\prime \prime \prime \prime}\right) X_{m} Y_{n} d x d y, \\
\left(Y_{7}, Y_{9}, Y_{11}, Y_{13}\right)= & \int_{0}^{a} \int_{0}^{b}\left(X_{m}^{\prime} Y_{n^{\prime}}^{\prime} X_{m}^{\prime \prime} Y_{n^{\prime}}\right. \\
& \left.X_{m}^{\prime \prime} Y_{n^{\prime}}^{\prime \prime}, X_{m}^{\prime \prime \prime \prime} Y_{n}\right) X_{m} Y_{n} d x d y
\end{aligned}
$$

\section{Numerical Validation}

\subsection{Comparative study}

Numerical study begins with the validation and appreciation of simple polynomial based plate theory (PolyF3) through flexural analysis along with other plate theories listed in Table 1. The second part of the study showcases smart application of piezoelectric material for shape control of FGM plate in which the simple polynomial $\left(F z=\frac{4 Z^{3}}{3 h_{t}^{2}}\right)$ based plate theory (PolyF3) is used.

In first part an FGM plate without considering piezoelectric layers made of metal $\left(E_{m}=70 \mathrm{GPa}\right)$ on one side and ceramic $\left(E_{c}=380 \mathrm{GPa}\right)$ on other side is examined. The value of Poisson's ratio is considered to be constant $\mu_{z}=0.3$. The load applied is sinusoidal with maximum ordinate $q_{0}$ at the center. The values of non-dimensional [59] transverse deflection, stresses used are as given in Table 4. Table 5 gives the results for transverse deflection of simply supported plate for change in power law and

Table 4 Non-dimensional deflection and stresses used in the study.

$$
\begin{array}{ll}
\bar{u}=\frac{100 E_{c} h^{3}}{q_{0} a^{4}} u\left(\frac{a}{2}, \frac{b}{2}, \frac{-h}{4}\right) & \bar{v}=\frac{100 E_{c} h^{3}}{q_{0} a^{4}} v\left(\frac{a}{2}, \frac{b}{2}, \frac{-h}{6}\right) \\
\bar{w}=\frac{10 E_{c} h^{3}}{q_{0} a^{4}} w\left(\frac{a}{2}, \frac{b}{2}, 0\right) & \bar{\sigma}_{x}=\frac{h}{q_{0} a} \sigma_{x}\left(\frac{a}{2}, \frac{b}{2}, \frac{h}{2}\right) \\
\bar{\sigma}_{y}=\frac{h}{q_{0} a} \sigma_{y}\left(\frac{a}{2}, \frac{b}{2}, \frac{h}{3}\right) & \bar{\sigma}_{y z}=\frac{h}{q_{0} a} \sigma_{y z}\left(0, \frac{b}{2}, \frac{h}{6}\right) \\
\bar{\sigma}_{x z}=\frac{h}{q_{0} a} \sigma_{x z}\left(\frac{a}{2}, 0,0\right) & \bar{\sigma}_{x y}=\frac{h}{q_{0} a} \sigma_{x y}\left(0,0, \frac{-h}{3}\right)
\end{array}
$$


Table 5 Non-dimensional deflection $\bar{w}$ of SSSS plate under sinusoidal loading.

\begin{tabular}{|c|c|c|c|c|}
\hline \multirow[t]{2}{*}{$\mathrm{N}$} & \multirow[t]{2}{*}{ Theory } & \multicolumn{3}{|l|}{$\bar{w}$} \\
\hline & & $\frac{a}{h}=4$ & $\frac{a}{h}=10$ & $\frac{a}{h}=100$ \\
\hline \multirow[t]{9}{*}{1} & CPT [60] & 0.5623 & 0.5623 & 0.5623 \\
\hline & FSDT [60] & 0.7291 & 0.5889 & 0.5625 \\
\hline & Quasi-3D [9] & 0.7289 & 0.5890 & 0.5625 \\
\hline & PolyF1 & 0.6949 & 0.5616 & 0.5365 \\
\hline & PolyF2 & 0.7023 & 0.5629 & 0.5365 \\
\hline & TrigF & 0.7019 & 0.5628 & 0.5365 \\
\hline & HyperF & 0.7024 & 0.5629 & 0.5365 \\
\hline & ExpF & 0.7010 & 0.5627 & 0.5365 \\
\hline & PolyF3 & 0.7023 & 0.5629 & 0.5365 \\
\hline \multirow[t]{9}{*}{4} & CPT [60] & 0.8281 & 0.8281 & 0.8281 \\
\hline & FSDT [60] & 1.1125 & 0.8736 & 0.8281 \\
\hline & Quasi-3D [9] & 1.1673 & 0.8828 & 0.8286 \\
\hline & PolyF1 & 1.0643 & 0.8156 & 0.7686 \\
\hline & PolyF2 & 1.1002 & 0.8215 & 0.7687 \\
\hline & TrigF & 1.1023 & 0.8219 & 0.7687 \\
\hline & HyperF & 1.1000 & 0.8215 & 0.7687 \\
\hline & ExpF & 1.1031 & 0.8221 & 0.7687 \\
\hline & PolyF3 & 1.1002 & 0.8215 & 0.7687 \\
\hline \multirow[t]{9}{*}{10} & CPT [60] & 0.9355 & 0.9355 & 0.9355 \\
\hline & FSDT [60] & 1.3178 & 0.9966 & 0.9360 \\
\hline & Quasi-3D [9] & 1.3925 & 1.0090 & 0.9361 \\
\hline & PolyF1 & 1.3056 & 0.9658 & 0.9016 \\
\hline & PolyF2 & 1.3551 & 0.9740 & 0.9017 \\
\hline & TrigF & 1.3559 & 0.9742 & 0.9017 \\
\hline & HyperF & 1.3550 & 0.9740 & 0.9017 \\
\hline & ExpF & 1.3547 & 0.9741 & 0.9017 \\
\hline & PolyF3 & 1.3551 & 0.9740 & 0.9017 \\
\hline
\end{tabular}

length to thickness aspect ratio. In second example complete stress profile for FGM plate simply supported on all edges with $a / h=10$ and variation in power law is analyzed whose results are given in Table 6 . The combination of simply supported and clamped edges through analytical solution based on beam functions is validated in third example for FGM plate with change in power law as well as thickness ratio as given in Tables 7, 8 and 9, also the results are compared with numerical FEM solution based on four variable shear deformation theory.

\subsection{Shape control}

The main part of the study is to demonstrate smart application of piezoelectric material members for shape control of FGM plate. Consider the square FGM plate coupled with piezoelectric layers of sides $200 \mathrm{~mm}$ with core of thickness $5 \mathrm{~mm}$ while piezoelectric layers of $0.1 \mathrm{~mm}$. The plate is subjected to uniform load of $100 \mathrm{~N} / \mathrm{m}^{2}$. The FGM core is made of Ti-6Al-4V (Titanium-alloy) and Aluminum oxide with piezoelectric layers of PZT-G1195N. The material properties for same can be found in [30]. The parametric study for variation in constant displacement gain feedback $G_{d}$ along with change in power law is performed whose results are given in Tables 10,11, 12 and 13. Figures 2, 3, 4 and 5 shows the smart application of piezoelectric material for shape control through closed loop feedback control law based on displacement along with possible combination of boundary conditions. All the results in this part are obtained with simple plate theory (PolyF3).

\section{Conclusion}

The principle objective of developing a simple mathematical model for bending (flexural) analysis and shape control of the FGM plate is revealed through the detailed procedure along with numerical study presented here. The simple plate model helps in the analysis of plate structures encompassing deflection checks, shape control as an application towards the structural health monitoring. The simple polynomial based plate theory (PolyF3) greatly breaks it down in terms of computation process and time required for analysis of the plate with ensured accuracy. Complete flexural analysis effectively maps the robustness and applicability of presented mathematical model. To handle the complex boundary conditions with a view to extend the approach current model, beam function comes to great aid as part of approximate solution. After performing the in depth analysis and achieving shape control of FGM plate with help of piezoelectric material following inferences are drawn working on the path of objectives initially defined;

- A mathematical model is presented with detailed formulation starting from the displacement field going up to governing equations with approximate analytical solution using beam functions satisfying the objective of study to develop simple mathematical model.

- Polynomial based shear deformation function (PolyF3) gives accurate results when compared with trigonometric, hyperbolic, exponential functions as demonstrated in Tables 5, 6, 7, 8 and 9. Bending study along with complete flexural analysis of FGM plate using simple mathematical model performed in first part of study explores its capability.

- The second important objective devised initially is to perform shape control of the FGM plate through smart application of piezoelectric material. In a closed loop system it is seen that increase in constant dis- 
Table 6 Dimensionless stress and deflections with change in power law $\frac{a}{h}=10$

\begin{tabular}{|c|c|c|c|c|c|c|c|c|c|}
\hline $\mathrm{N}$ & Theory & $\bar{u}\left(\frac{-h}{4}\right)$ & $\bar{u}\left(\frac{-h}{6}\right)$ & $\bar{w}(0)$ & $\bar{\sigma}_{x}\left(\frac{h}{2}\right)$ & $\bar{\sigma}_{y}\left(\frac{h}{3}\right)$ & $\bar{\sigma}_{y z}\left(\frac{h}{\sigma}\right)$ & $\bar{\sigma}_{y z}(0)$ & $\bar{\sigma}_{x y}\left(\frac{-h}{3}\right)$ \\
\hline \multirow[t]{8}{*}{ Ceramic } & GSDT [59] & 0.2309 & 0.1539 & 0.2960 & 1.9955 & 1.3121 & 0.2132 & 0.2462 & 0.7065 \\
\hline & Quasi-3D [61] & 0.2063 & 0.1369 & 0.2881 & 2.0645 & 1.3456 & 0.2963 & 0.3335 & 0.6689 \\
\hline & PolyF1 & 0.2193 & 0.1459 & 0.2953 & 1.9846 & 1.3149 & 0.1920 & 0.2046 & 0.7080 \\
\hline & PolyF2 & 0.2183 & 0.1451 & 0.2961 & 1.9943 & 1.3124 & 0.2121 & 0.2386 & 0.7067 \\
\hline & TrigF & 0.2181 & 0.1448 & 0.2960 & 1.9955 & 1.3121 & 0.2132 & 0.2462 & 0.7065 \\
\hline & HyperF & 0.2183 & 0.1451 & 0.2961 & 1.9942 & 1.3124 & 0.2119 & 0.2379 & 0.7067 \\
\hline & ExpF & 0.2179 & 0.1446 & 0.2960 & 1.9966 & 1.3119 & 0.2134 & 0.2538 & 0.7064 \\
\hline & PolyF3 & 0.2183 & 0.1451 & 0.2961 & 1.9943 & 1.3124 & 0.2121 & 0.2386 & 0.7067 \\
\hline \multirow[t]{8}{*}{1} & GSDT [59] & 0.6626 & 0.5093 & 0.5889 & 3.0870 & 1.4894 & 0.2622 & 0.2462 & 0.6110 \\
\hline & Quasi-3D [61] & 0.571 & 0.4348 & 0.5592 & 3.1756 & 1.4962 & 0.3644 & 0.3335 & 0.5486 \\
\hline & PolyF1 & 0.6430 & 0.4959 & 0.5877 & 3.0686 & 1.4935 & 0.2360 & 0.2046 & 0.6118 \\
\hline & PolyF2 & 0.6414 & 0.4944 & 0.5890 & 3.0850 & 1.4898 & 0.2608 & 0.2386 & 0.6111 \\
\hline & TrigF & 0.6410 & 0.4940 & 0.5889 & 3.0870 & 1.4894 & 0.2622 & 0.2462 & 0.6110 \\
\hline & HyperF & 0.6414 & 0.4944 & 0.5890 & 3.0848 & 1.4898 & 0.2606 & 0.2379 & 0.6111 \\
\hline & ExpF & 0.6407 & 0.4935 & 0.5888 & 3.0889 & 1.4891 & 0.2624 & 0.2538 & 0.6110 \\
\hline & PolyF3 & 0.6414 & 0.4944 & 0.5890 & 3.0850 & 1.4898 & 0.2608 & 0.2386 & 0.6111 \\
\hline \multirow[t]{8}{*}{2} & GSDT [59] & 0.9281 & 0.7311 & 0.7573 & 3.6094 & 1.3954 & 0.2763 & 0.2265 & 0.5441 \\
\hline & Quasi-3D [61] & 0.7941 & 0.6197 & 0.7158 & 3.6833 & 1.3775 & 0.3502 & 0.2797 & 0.4853 \\
\hline & PolyF1 & 0.9010 & 0.7126 & 0.7547 & 3.5846 & 1.4012 & 0.2401 & 0.1816 & 0.5451 \\
\hline & PolyF2 & 0.8984 & 0.7101 & 0.7573 & 3.6067 & 1.3960 & 0.2737 & 0.2186 & 0.5442 \\
\hline & TrigF & 0.8978 & 0.7095 & 0.7573 & 3.6094 & 1.3954 & 0.2763 & 0.2265 & 0.5441 \\
\hline & HyperF & 0.8984 & 0.7102 & 0.7573 & 3.6064 & 1.3961 & 0.2734 & 0.2178 & 0.5442 \\
\hline & ExpF & 0.8972 & 0.7088 & 0.7572 & 3.6119 & 1.3949 & 0.2777 & 0.2345 & 0.5440 \\
\hline & PolyF3 & 0.8984 & 0.7101 & 0.7573 & 3.6067 & 1.3960 & 0.2737 & 0.2186 & 0.5442 \\
\hline \multirow[t]{8}{*}{3} & GSDT [59] & 1.0447 & 0.8271 & 0.8377 & 3.8742 & 1.2748 & 0.2715 & 0.2107 & 0.5525 \\
\hline & Quasi-3D [61] & 0.8527 & 0.2539 & 0.7944 & 3.9224 & 0.4846 & 0.3175 & 0.2401 & 7.1292 \\
\hline & PolyF1 & 1.0109 & 0.8039 & 0.8332 & 3.8437 & 1.2820 & 0.2279 & 0.1632 & 0.5538 \\
\hline & PolyF2 & 1.0073 & 0.8007 & 0.8375 & 3.8709 & 1.2756 & 0.2677 & 0.2024 & 0.5526 \\
\hline & TrigF & 1.0065 & 0.7998 & 0.8377 & 3.8742 & 1.2748 & 0.2715 & 0.2107 & 0.5525 \\
\hline & HyperF & 1.0074 & 0.8008 & 0.8374 & 3.8706 & 1.2757 & 0.2673 & 0.2016 & 0.5527 \\
\hline & ExpF & 1.0057 & 0.7989 & 0.8377 & 3.8773 & 1.2740 & 0.2741 & 0.2190 & 0.5523 \\
\hline & PolyF3 & 1.0073 & 0.8007 & 0.8375 & 3.8709 & 1.2756 & 0.2677 & 0.2024 & 0.5526 \\
\hline \multirow[t]{8}{*}{5} & GSDT [59] & 1.1158 & 0.8792 & 0.9118 & 4.2488 & 1.1029 & 0.2429 & 0.2017 & 0.5755 \\
\hline & Quasi-3D [61] & 0.9567 & 0.7464 & 0.8729 & 4.2607 & 1.0651 & 0.2607 & 0.211 & 0.5213 \\
\hline & PolyF1 & 1.0716 & 0.8489 & 0.9042 & 4.2108 & 1.1117 & 0.1966 & 0.1507 & 0.5771 \\
\hline & PolyF2 & 1.0669 & 0.8448 & 0.9114 & 4.2447 & 1.1041 & 0.2385 & 0.1930 & 0.5757 \\
\hline & TrigF & 1.0659 & 0.8437 & 0.9118 & 4.2488 & 1.1029 & 0.2429 & 0.2017 & 0.5755 \\
\hline & HyperF & 1.0670 & 0.8449 & 0.9113 & 4.2443 & 1.1042 & 0.2381 & 0.1922 & 0.5757 \\
\hline & ExpF & 1.0648 & 0.8425 & 0.9121 & 4.2527 & 1.1019 & 0.2461 & 0.2105 & 0.5753 \\
\hline & PolyF3 & 1.0669 & 0.8448 & 0.9114 & 4.2447 & 1.1041 & 0.2385 & 0.1930 & 0.5757 \\
\hline \multirow[t]{8}{*}{10} & GSDT [59] & 1.1372 & 0.8756 & 1.0089 & 5.0890 & 0.8775 & 0.2041 & 0.2198 & 0.5894 \\
\hline & Quasi-3D [61] & 0.9841 & 0.7498 & 0.9806 & 5.0901 & 0.853 & 0.2173 & 0.2281 & 0.5442 \\
\hline & PolyF1 & 1.0820 & 0.8376 & 1.0004 & 5.0468 & 0.8854 & 0.1668 & 0.1658 & 0.5913 \\
\hline & PolyF2 & 1.0766 & 0.8329 & 1.0087 & 5.0849 & 0.8785 & 0.2014 & 0.2114 & 0.5896 \\
\hline & TrigF & 1.0754 & 0.8317 & 1.0089 & 5.0890 & 0.8775 & 0.2041 & 0.2198 & 0.5894 \\
\hline & HyperF & 1.0767 & 0.8330 & 1.0087 & 5.0845 & 0.8786 & 0.2011 & 0.2106 & 0.5896 \\
\hline & ExpF & 1.0743 & 0.8303 & 1.0088 & 5.0928 & 0.8766 & 0.2057 & 0.2282 & 0.5892 \\
\hline & PolyF3 & 1.0766 & 0.8329 & 1.0087 & 5.0849 & 0.8785 & 0.2014 & 0.2114 & 0.5896 \\
\hline
\end{tabular}


Table 6 (continued)

\begin{tabular}{llllllllll}
\hline $\mathrm{N}$ & Theory & $\bar{u}\left(\frac{-h}{4}\right)$ & $\bar{u}\left(\frac{-h}{6}\right)$ & $\bar{w}(0)$ & $\bar{\sigma}_{x}\left(\frac{h}{2}\right)$ & $\bar{\sigma}_{y}\left(\frac{h}{3}\right)$ & $\bar{\sigma}_{y z}\left(\frac{h}{6}\right)$ & $\bar{\sigma}_{y z}(0)$ & $\bar{\sigma}_{x y}\left(\frac{-h}{3}\right)$ \\
\hline Metal & GSDT [59] & 1.2534 & 0.8356 & 1.6070 & 1.9955 & 1.3121 & 0.2132 & 0.2462 & 0.7065 \\
& Quasi-3D [61] & 1.1199 & 0.7432 & 1.5642 & 2.0645 & 1.3456 & 0.2963 & 0.3335 & 0.6689 \\
& PolyF1 & 1.1851 & 0.7923 & 1.5831 & 10.5868 & 1.2891 & 0.1915 & 0.2041 & 0.7034 \\
& PolyF2 & 1.1799 & 0.7875 & 1.5870 & 10.6385 & 1.2865 & 0.2116 & 0.2381 & 0.7020 \\
& TrigF & 1.1424 & 0.7880 & 1.4396 & 9.2637 & 1.0953 & 0.2094 & 0.2418 & 0.6687 \\
& HyperF & 1.1437 & 0.7894 & 1.4399 & 9.2580 & 1.0958 & 0.2084 & 0.2339 & 0.6688 \\
& ExpF & 1.1413 & 0.7867 & 1.4390 & 9.2687 & 1.0950 & 0.2094 & 0.2490 & 0.6685 \\
& PolyF3 & 1.1436 & 0.7893 & 1.4399 & 9.2585 & 1.0957 & 0.2085 & 0.2346 & 0.6688 \\
\hline
\end{tabular}

placement feedback gain $G_{d}$ in case of Ti- $6 \mathrm{Al}-4 \mathrm{~V}$ and $\mathrm{Al}_{2} \mathrm{O}_{3} \mathrm{FGM}$ plate decreases the transverse displacement as shown in Figs. 2, 3, 4 and 5 for a combination of clamped and simply supported boundary conditions.

- Tabulated numerical results for control of deflection with change in $G_{d}$, boundary conditions and power law are given in Tables 10,11, 12 and 13 for the Ti-6Al-4V and $\mathrm{Al}_{2} \mathrm{O}_{3} \mathrm{FGM}$ plate, with a view to reinforce the strength of plate model in case of variation in input properties.

- The present model successfully demonstrates its robustness in bending analysis along with the smart application of piezoelectric material to achieve shape control of FGM plate following a simple analytical approach compared to a complex numerical solution.
- Though the current model is complete in all aspects of mathematical terms still the practical application of such concept is under progress. The cost of material especially piezoelectric is big lacking point for large scale application of practical study or actual physical use.

- Another limitation of present study is the approximate analysis approach instead of complete three dimensional elastic solution. The beam function provides the approximate analytical solution based on satisfaction of geometric boundary condition.

- Scope exists for the application of the presented governing system to perform vibration, buckling analysis along with active control and stability study on FGM plate with piezoelectric material.

- The present model can extend its reach to partial three dimensional elastic analysis of plates by incorporating the thickness stretching effect such that strain in transverse (z-axis) is not equal to zero. 
Table 7 Non-dimensional value of deflection $\bar{w}$ for $\frac{a}{h}=5$

\begin{tabular}{|c|c|c|c|c|c|}
\hline \multirow[t]{2}{*}{$N$} & \multirow[t]{2}{*}{ Theory } & \multicolumn{4}{|l|}{$\bar{w}$} \\
\hline & & $\mathrm{CCCC}$ & SCSC & SSSC & SSSS \\
\hline \multirow[t]{7}{*}{0} & FEM [47] & 0.2356 & 0.3090 & 0.4020 & 0.5381 \\
\hline & PolyF1 & 0.2266 & 0.3050 & 0.3951 & 0.5519 \\
\hline & PolyF2 & 0.2303 & 0.3091 & 0.3992 & 0.5566 \\
\hline & TrigF & 0.2300 & 0.3088 & 0.3990 & 0.5564 \\
\hline & HyperF & 0.2303 & 0.3091 & 0.3992 & 0.5566 \\
\hline & ExpF & 0.2294 & 0.3083 & 0.3985 & 0.5558 \\
\hline & PolyF3 & 0.2303 & 0.3091 & 0.3992 & 0.5566 \\
\hline \multirow[t]{7}{*}{0.5} & FEM [47] & 0.3452 & 0.4577 & 0.6015 & 0.8120 \\
\hline & PolyF1 & 0.3314 & 0.4486 & 0.5834 & 0.8201 \\
\hline & PolyF2 & 0.3358 & 0.4536 & 0.5884 & 0.8257 \\
\hline & TrigF & 0.3355 & 0.4532 & 0.5881 & 0.8254 \\
\hline & HyperF & 0.3358 & 0.4536 & 0.5884 & 0.8257 \\
\hline & ExpF & 0.3347 & 0.4525 & 0.5874 & 0.8247 \\
\hline & PolyF3 & 0.3358 & 0.4536 & 0.5884 & 0.8257 \\
\hline \multirow[t]{7}{*}{1} & FEM [47] & 0.4397 & 0.5857 & 0.7734 & 1.0486 \\
\hline & PolyF1 & 0.4189 & 0.5662 & 0.7335 & 1.0340 \\
\hline & PolyF2 & 0.4252 & 0.5731 & 0.7405 & 1.0419 \\
\hline & TrigF & 0.4247 & 0.5727 & 0.7401 & 1.0415 \\
\hline & HyperF & 0.4252 & 0.5731 & 0.7405 & 1.0419 \\
\hline & ExpF & 0.4238 & 0.5718 & 0.7393 & 1.0406 \\
\hline & PolyF3 & 0.4252 & 0.5731 & 0.7405 & 1.0419 \\
\hline \multirow[t]{7}{*}{2} & FEM [47] & 0.5739 & 0.7610 & 1.0028 & 1.3572 \\
\hline & PolyF1 & 0.5384 & 0.7215 & 0.9251 & 1.3024 \\
\hline & PolyF2 & 0.5515 & 0.7360 & 0.9397 & 1.3188 \\
\hline & TrigF & 0.5514 & 0.7360 & 0.9398 & 1.3190 \\
\hline & HyperF & 0.5515 & 0.7360 & 0.9397 & 1.3188 \\
\hline & ExpF & 0.5505 & 0.7353 & 0.9392 & 1.3184 \\
\hline & PolyF3 & 0.5515 & 0.7360 & 0.9397 & 1.3188 \\
\hline \multirow[t]{7}{*}{5} & FEM [47] & 0.7649 & 0.9896 & 1.2770 & 1.6973 \\
\hline & PolyF1 & 0.7043 & 0.9283 & 1.1721 & 1.6295 \\
\hline & PolyF2 & 0.7413 & 0.9689 & 1.2125 & 1.6748 \\
\hline & TrigF & 0.7433 & 0.9712 & 1.2150 & 1.6777 \\
\hline & HyperF & 0.7411 & 0.9686 & 1.2122 & 1.6745 \\
\hline & ExpF & 0.7439 & 0.9722 & 1.2162 & 1.6791 \\
\hline & PolyF3 & 0.7413 & 0.9689 & 1.2125 & 1.6748 \\
\hline \multirow[t]{7}{*}{10} & FEM [47] & 0.8876 & 1.1365 & 1.4518 & 1.9125 \\
\hline & PolyF1 & 0.8247 & 1.0818 & 1.3622 & 1.8806 \\
\hline & PolyF2 & 0.8673 & 1.1285 & 1.4085 & 1.9329 \\
\hline & TrigF & 0.8676 & 1.1291 & 1.4096 & 1.9338 \\
\hline & HyperF & 0.8672 & 1.1284 & 1.4083 & 1.9327 \\
\hline & ExpF & 0.8661 & 1.1278 & 1.4085 & 1.9328 \\
\hline & PolyF3 & 0.8673 & 1.1285 & 1.4085 & 1.9329 \\
\hline
\end{tabular}

Table 8 Non-dimensional value of deflection $\bar{w}$ for $\frac{a}{h}=10$

\begin{tabular}{|c|c|c|c|c|c|}
\hline \multirow[t]{2}{*}{$\mathrm{N}$} & \multirow[t]{2}{*}{ Theory } & \multicolumn{4}{|l|}{$\bar{w}$} \\
\hline & & $\mathrm{CCCC}$ & SCSC & SSSC & SSSS \\
\hline \multirow[t]{7}{*}{0} & FEM [47] & 0.1709 & 0.2424 & 0.3343 & 0.4693 \\
\hline & PolyF1 & 0.1642 & 0.2379 & 0.3292 & 0.4787 \\
\hline & PolyF2 & 0.1652 & 0.2390 & 0.3303 & 0.4799 \\
\hline & TrigF & 0.1652 & 0.2389 & 0.3302 & 0.4799 \\
\hline & HyperF & 0.1652 & 0.2390 & 0.3303 & 0.4799 \\
\hline & ExpF & 0.1650 & 0.2388 & 0.3301 & 0.4799 \\
\hline & PolyF3 & 0.1652 & 0.2390 & 0.3303 & 0.4799 \\
\hline \multirow[t]{7}{*}{0.5} & FEM [47] & 0.2577 & 0.3676 & 0.5099 & 0.7190 \\
\hline & PolyF1 & 0.2466 & 0.3574 & 0.4939 & 0.7207 \\
\hline & PolyF2 & 0.2478 & 0.3587 & 0.4952 & 0.7221 \\
\hline & TrigF & 0.2477 & 0.3586 & 0.4951 & 0.7221 \\
\hline & HyperF & 0.2478 & 0.3587 & 0.4952 & 0.7221 \\
\hline & ExpF & 0.2476 & 0.3585 & 0.4950 & 0.7221 \\
\hline & PolyF3 & 0.2478 & 0.3587 & 0.4952 & 0.7221 \\
\hline \multirow[t]{7}{*}{1} & FEM [47] & 0.3306 & 0.4732 & 0.6591 & 0.9324 \\
\hline & PolyF1 & 0.3136 & 0.4528 & 0.6222 & 0.9105 \\
\hline & PolyF2 & 0.3153 & 0.4547 & 0.6240 & 0.9125 \\
\hline & TrigF & 0.3152 & 0.4546 & 0.6240 & 0.9124 \\
\hline & HyperF & 0.3153 & 0.4547 & 0.6240 & 0.9125 \\
\hline & ExpF & 0.3150 & 0.4544 & 0.6238 & 0.9122 \\
\hline & PolyF3 & 0.3153 & 0.4547 & 0.6240 & 0.9125 \\
\hline \multirow[t]{7}{*}{2} & FEM [47] & 0.4237 & 0.6061 & 0.8454 & 1.1973 \\
\hline & PolyF1 & 0.3967 & 0.5690 & 0.7753 & 1.1362 \\
\hline & PolyF2 & 0.4002 & 0.5728 & 0.7791 & 1.1404 \\
\hline & TrigF & 0.4002 & 0.5729 & 0.7792 & 1.1405 \\
\hline & HyperF & 0.4002 & 0.5728 & 0.7791 & 1.1404 \\
\hline & ExpF & 0.4001 & 0.5728 & 0.7791 & 1.1403 \\
\hline & PolyF3 & 0.4002 & 0.5728 & 0.7791 & 1.1404 \\
\hline \multirow[t]{7}{*}{5} & FEM [47] & 0.5241 & 0.7406 & 1.0235 & 1.4393 \\
\hline & PolyF1 & 0.4883 & 0.6959 & 0.9553 & 1.3761 \\
\hline & PolyF2 & 0.4981 & 0.7064 & 0.9542 & 1.3876 \\
\hline & TrigF & 0.4987 & 0.7071 & 0.9549 & 1.3884 \\
\hline & HyperF & 0.4980 & 0.7064 & 0.9542 & 1.3876 \\
\hline & ExpF & 0.4990 & 0.7075 & 0.9593 & 1.3888 \\
\hline & PolyF3 & 0.4981 & 0.7064 & 0.9542 & 1.3876 \\
\hline \multirow[t]{7}{*}{10} & FEM [47] & 0.5911 & 0.8297 & 1.1391 & 1.5937 \\
\hline & PolyF1 & 0.5561 & 0.7928 & 1.0786 & 1.5656 \\
\hline & PolyF2 & 0.5674 & 0.8050 & 1.0906 & 1.5790 \\
\hline & TrigF & 0.5676 & 0.8053 & 1.0909 & 1.5793 \\
\hline & HyperF & 0.5673 & 0.8050 & 1.0906 & 1.5790 \\
\hline & ExpF & 0.5675 & 0.8051 & 1.0907 & 1.5791 \\
\hline & PolyF3 & 0.5674 & 0.8050 & 1.0906 & 1.5790 \\
\hline
\end{tabular}


Table 9 Non-dimensional value of deflection $\bar{w}$ for $\frac{a}{h}=100$

\begin{tabular}{|c|c|c|c|c|c|}
\hline \multirow[t]{2}{*}{$\mathrm{N}$} & \multirow[t]{2}{*}{ Theory } & \multicolumn{4}{|l|}{$\bar{W}$} \\
\hline & & CCCC & SCSC & SSSC & SSSS \\
\hline \multirow[t]{7}{*}{0} & FEM [47] & 0.1493 & 0.2202 & 0.3118 & 0.4465 \\
\hline & PolyF1 & 0.1436 & 0.2157 & 0.3074 & 0.4546 \\
\hline & PolyF2 & 0.1436 & 0.2157 & 0.3074 & 0.4546 \\
\hline & TrigF & 0.1436 & 0.2157 & 0.3074 & 0.4546 \\
\hline & HyperF & 0.1436 & 0.2157 & 0.3074 & 0.4546 \\
\hline & ExpF & 0.1436 & 0.2157 & 0.3074 & 0.4546 \\
\hline & PolyF3 & 0.1436 & 0.2157 & 0.3074 & 0.4546 \\
\hline \multirow[t]{7}{*}{0.5} & FEM [47] & 0.2284 & 0.3376 & 0.4795 & 0.6882 \\
\hline & PolyF1 & 0.2158 & 0.3272 & 0.4643 & 0.6878 \\
\hline & PolyF2 & 0.2185 & 0.3273 & 0.4643 & 0.6878 \\
\hline & TrigF & 0.2185 & 0.3273 & 0.4643 & 0.6878 \\
\hline & HyperF & 0.2185 & 0.3273 & 0.4643 & 0.6878 \\
\hline & ExpF & 0.2185 & 0.3273 & 0.4643 & 0.6878 \\
\hline & PolyF3 & 0.2185 & 0.3273 & 0.4643 & 0.6878 \\
\hline \multirow[t]{7}{*}{1} & FEM [47] & 0.2940 & 0.4357 & 0.6211 & 0.8940 \\
\hline & PolyF1 & 0.2787 & 0.4154 & 0.5854 & 0.8697 \\
\hline & PolyF2 & 0.2788 & 0.4154 & 0.5855 & 0.8697 \\
\hline & TrigF & 0.2788 & 0.4154 & 0.5855 & 0.8697 \\
\hline & HyperF & 0.2788 & 0.4154 & 0.5855 & 0.8697 \\
\hline & ExpF & 0.2788 & 0.4154 & 0.5855 & 0.8697 \\
\hline & PolyF3 & 0.2788 & 0.4154 & 0.5855 & 0.8697 \\
\hline \multirow[t]{7}{*}{2} & FEM [47] & 0.3732 & 0.5545 & 0.7931 & 1.1444 \\
\hline & PolyF1 & 0.3498 & 0.5186 & 0.7259 & 1.0813 \\
\hline & PolyF2 & 0.3498 & 0.5187 & 0.7259 & 1.0813 \\
\hline & TrigF & 0.3498 & 0.5187 & 0.7259 & 1.0813 \\
\hline & HyperF & 0.3498 & 0.5187 & 0.7259 & 1.0813 \\
\hline & ExpF & 0.3498 & 0.5187 & 0.7259 & 1.0813 \\
\hline & PolyF3 & 0.3498 & 0.5187 & 0.7259 & 1.0813 \\
\hline \multirow[t]{7}{*}{5} & FEM [47] & 0.4430 & 0.6574 & 0.9392 & 1.3539 \\
\hline & PolyF1 & 0.4169 & 0.6191 & 0.8685 & 1.2924 \\
\hline & PolyF2 & 0.4170 & 0.6192 & 0.8686 & 1.2925 \\
\hline & TrigF & 0.4170 & 0.6192 & 0.8686 & 1.2925 \\
\hline & HyperF & 0.4170 & 0.6192 & 0.8686 & 1.2925 \\
\hline & ExpF & 0.4170 & 0.6192 & 0.8686 & 1.2925 \\
\hline & PolyF3 & 0.4170 & 0.6192 & 0.8686 & 1.2925 \\
\hline \multirow[t]{7}{*}{10} & FEM [47] & 0.4913 & 0.7272 & 1.0352 & 1.4883 \\
\hline & PolyF1 & 0.4672 & 0.6973 & 0.9849 & 1.4614 \\
\hline & PolyF2 & 0.4673 & 0.6974 & 0.9850 & 1.4618 \\
\hline & TrigF & 0.4673 & 0.6974 & 0.9850 & 1.4618 \\
\hline & HyperF & 0.4673 & 0.6974 & 0.9850 & 1.4618 \\
\hline & ExpF & 0.4673 & 0.6974 & 0.9850 & 1.4618 \\
\hline & PolyF3 & 0.4673 & 0.6974 & 0.9850 & 1.4618 \\
\hline
\end{tabular}

Table 10 Deflection $(\mathrm{m})$ of square SSSS FGM plate

\begin{tabular}{lllll}
\hline $\mathrm{N}$ & $\overline{\mathrm{w}} * 10^{-6}$ & & & \\
\cline { 2 - 5 } & $\mathrm{Gd}=1$ & $\mathrm{Gd}=5$ & $\mathrm{Gd}=10$ & $\mathrm{Gd}=30$ \\
\hline Ceramic & 8.1036 & 7.1764 & 6.2751 & 4.1696 \\
1 & 4.0358 & 3.7912 & 3.5238 & 2.7453 \\
2 & 3.7236 & 3.5144 & 3.2834 & 2.5971 \\
3 & 3.6084 & 3.4116 & 3.1935 & 2.5406 \\
5 & 3.4697 & 3.2874 & 3.0844 & 2.4710 \\
10 & 3.2732 & 3.1105 & 2.9281 & 2.3697 \\
Metal & 2.8784 & 2.7517 & 2.6081 & 2.1556 \\
\hline
\end{tabular}

Table 11 Deflection (m) of square CCCC FGM plate.

\begin{tabular}{lllll}
\hline $\mathrm{N}$ & $\bar{w} * 10^{-6}$ & & & \\
\cline { 2 - 5 } & $\mathrm{Gd}=1$ & $\mathrm{Gd}=5$ & $\mathrm{Gd}=10$ & $\mathrm{Gd}=30$ \\
\hline Ceramic & 2.6510 & 2.4980 & 2.3296 & 1.8323 \\
1 & 1.2953 & 1.2577 & 1.2135 & 1.0632 \\
2 & 1.1967 & 1.1645 & 1.1265 & 0.9958 \\
3 & 1.1620 & 1.1316 & 1.0957 & 0.9716 \\
5 & 1.1199 & 1.0917 & 1.0582 & 0.9420 \\
10 & 1.0583 & 1.0331 & 1.0031 & 0.8980 \\
Metal & 0.9308 & 0.9113 & 0.8878 & 0.8046 \\
\hline
\end{tabular}

Table 12 Deflection (m) of square SCSC FGM plate.

\begin{tabular}{lllll}
\hline $\mathrm{N}$ & $\bar{w} * 10^{-6}$ & & & \\
\cline { 2 - 5 } & $\mathrm{Gd}=1$ & $\mathrm{Gd}=5$ & $\mathrm{Gd}=10$ & $\mathrm{Gd}=30$ \\
\hline Ceramic & 3.9484 & 3.6669 & 3.3662 & 2.5312 \\
1 & 1.9353 & 1.8651 & 1.7841 & 1.5186 \\
2 & 1.7879 & 1.7278 & 1.6580 & 1.4263 \\
3 & 1.7357 & 1.6790 & 1.6130 & 1.3928 \\
5 & 1.6725 & 1.6198 & 1.5583 & 1.3518 \\
10 & 1.5803 & 1.5332 & 1.4780 & 1.2909 \\
Metal & 1.3900 & 1.3534 & 1.3102 & 1.1611 \\
\hline
\end{tabular}

Table 13 Deflection $(m)$ of square SSSC FGM plate.

\begin{tabular}{lllll}
\hline $\mathrm{N}$ & $\bar{W} * 10^{-6}$ & & & \\
\cline { 2 - 5 } & $\mathrm{Gd}=1$ & $\mathrm{Gd}=5$ & $\mathrm{Gd}=10$ & $\mathrm{Gd}=30$ \\
\hline Ceramic & 5.5153 & 4.9868 & 4.4525 & 3.1115 \\
1 & 2.7228 & 2.5874 & 2.4357 & 1.9710 \\
2 & 2.5144 & 2.3985 & 2.2676 & 1.8594 \\
3 & 2.4394 & 2.3301 & 2.2064 & 1.8181 \\
5 & 2.3487 & 2.2473 & 2.1320 & 1.7672 \\
10 & 2.2180 & 2.1273 & 2.0237 & 1.6922 \\
Metal & 1.9509 & 1.8804 & 1.7990 & 1.5322 \\
\hline
\end{tabular}



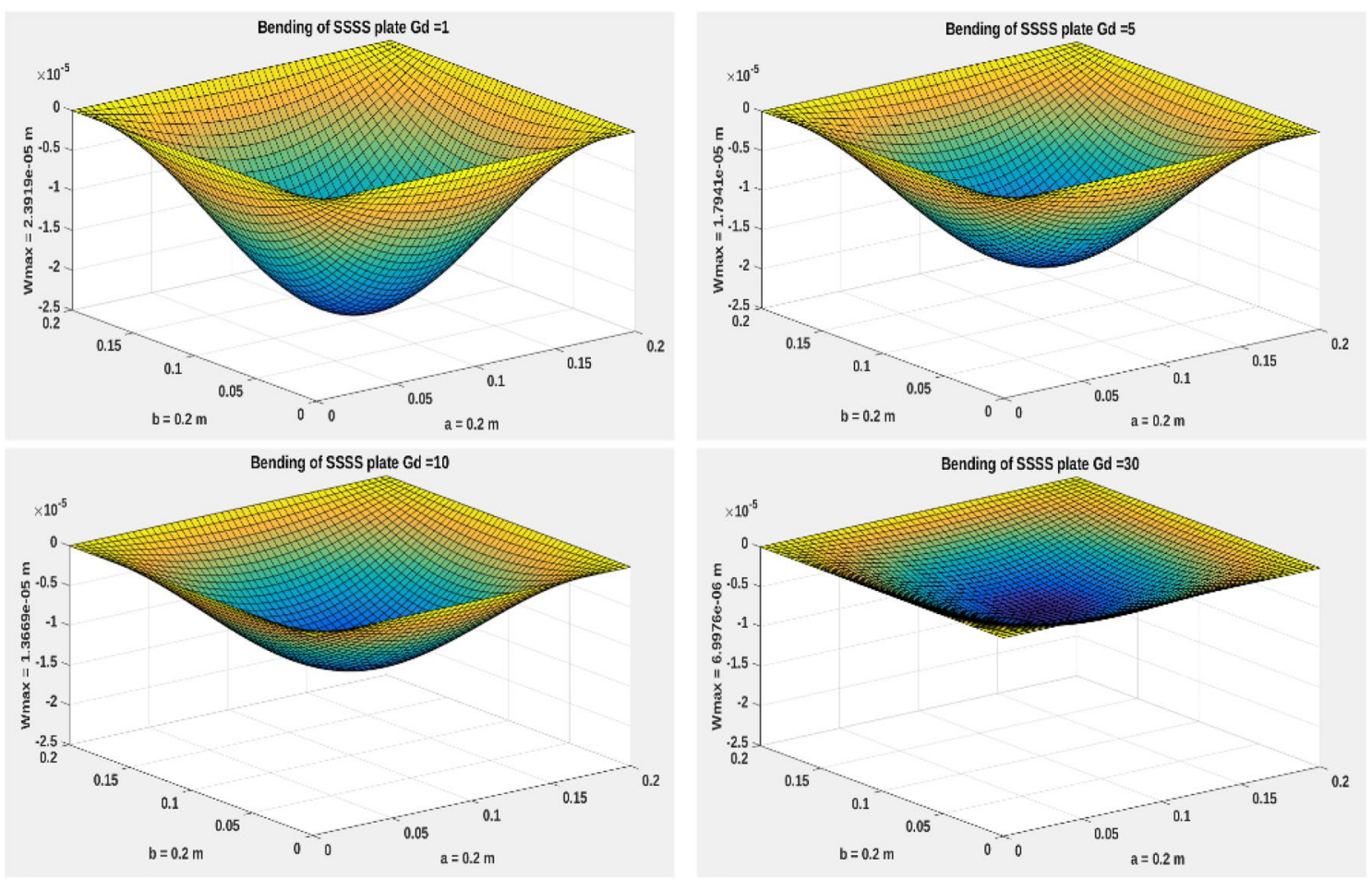

Fig. 2 Shape control of square SSSS FGM plate
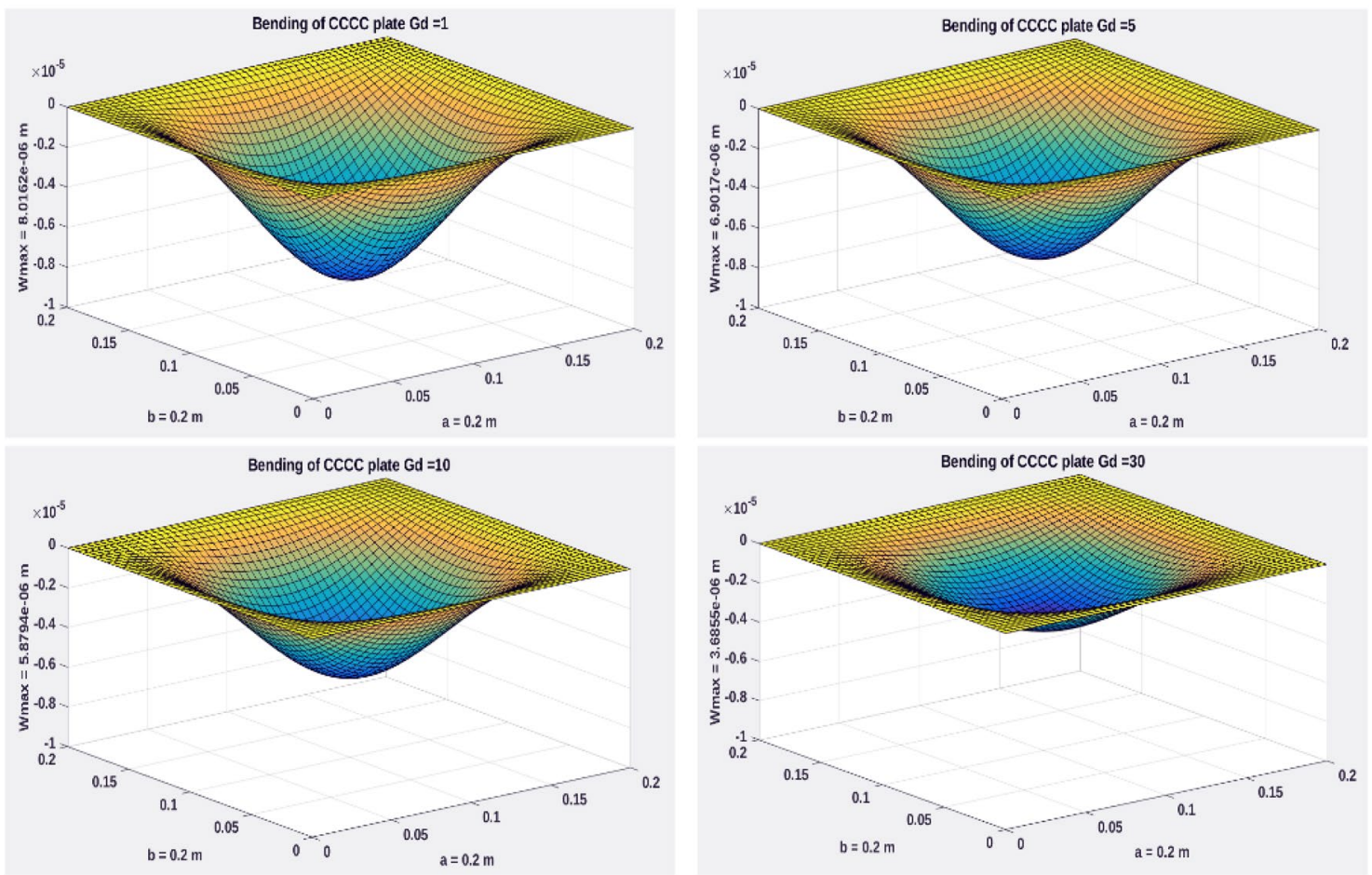

Fig. 3 Shape control of square CCCC FGM plate

\section{SN Applied Sciences}

A SPRINGER NATURE journal 

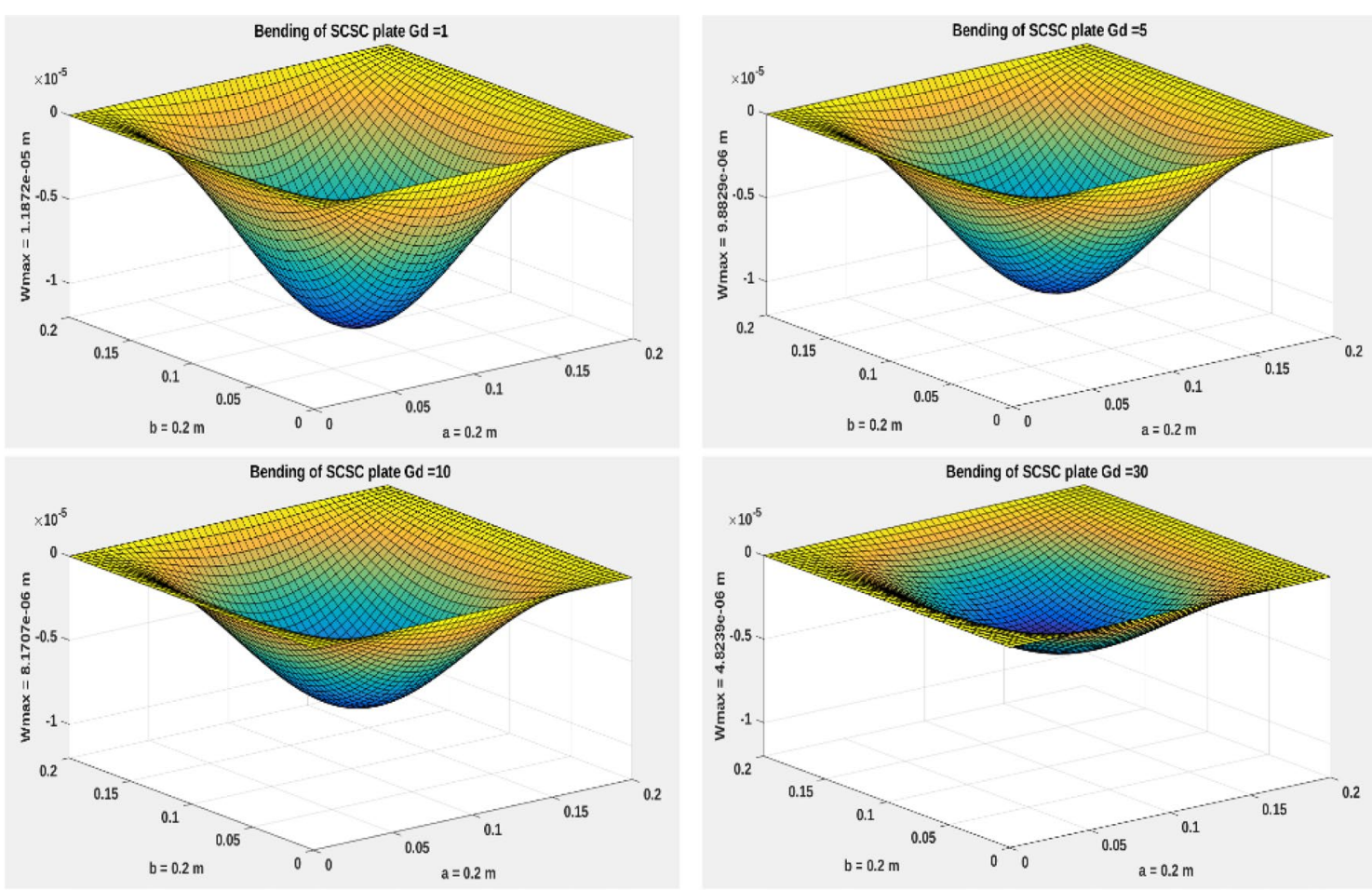

Fig. 4 Shape control of square SCSC FGM plate
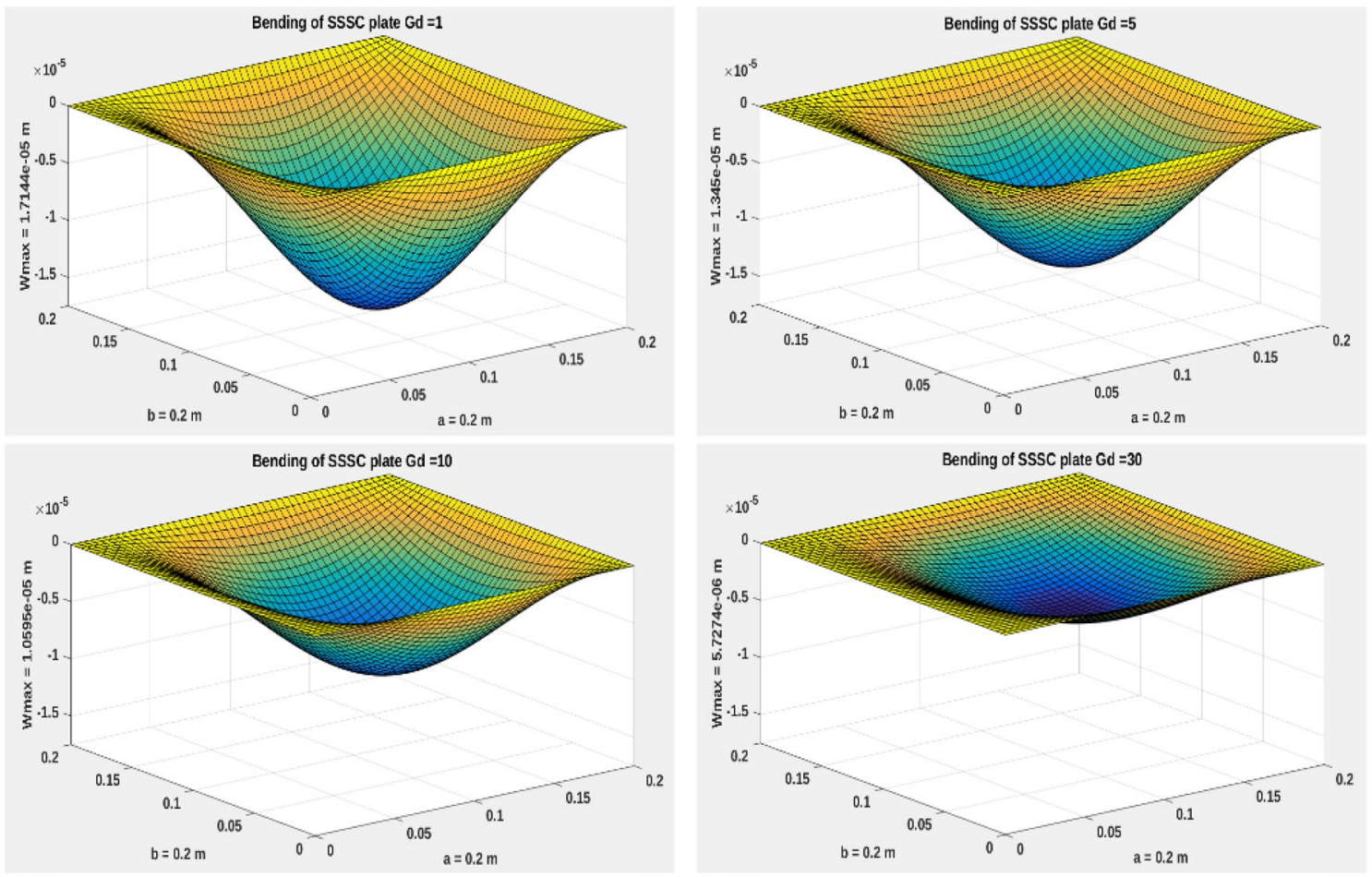

Fig. 5 shape control of square SSSC FGM plate 


\section{Compliance with ethical standards}

Conflict of interest The authors declare that they have no conflict of interest.

Open Access This article is licensed under a Creative Commons Attribution 4.0 International License, which permits use, sharing, adaptation, distribution and reproduction in any medium or format, as long as you give appropriate credit to the original author(s) and the source, provide a link to the Creative Commons licence, and indicate if changes were made. The images or other third party material in this article are included in the article's Creative Commons licence, unless indicated otherwise in a credit line to the material. If material is not included in the article's Creative Commons licence and your intended use is not permitted by statutory regulation or exceeds the permitted use, you will need to obtain permission directly from the copyright holder. To view a copy of this licence, visit http://creativecommons .org/licenses/by/4.0/.

\section{References}

1. Abad F, Rouzegar J (2017) An exact spectral element method for free vibration analysis of FG plate integrated with piezoelectric layers. Compos Struct 180:696-708

2. Alibeigloo A (2010) Three-dimensional exact solution for functionally graded rectangular plate with integrated surface piezoelectric layers resting on elastic foundation. Mech Adv Mater Struct 17(3):183-195

3. Behjat B, Khoshravan MR (2012) Nonlinear analysis of functionally graded laminates considering piezoelectric effect. J Mech Sci Technol 26(8):2581-2588

4. Bendine K, Wankhade RL (2017) Optimal shape control of piezolaminated beams with different boundary condition and loading using genetic algorithm. Int J Adv Struct Eng 9(4):375-384

5. Beslin O, Nicolas J (1997) A hierarchical functions set for predicting very high order plate bending modes with any boundary conditions. J Sound Vib 202(5):633-655

6. Bhat $R$ (1985) Natural frequencies of rectangular plates using characteristic orthogonal polynomials in Rayleigh-Ritz method. J Sound Vib 102(4):493-499

7. Bian Z, Ying J, Chen W, Ding H (2006) Bending and free vibration analysis of a smart functionally graded plate. Struct Eng Mech 23(1):97-113

8. Blevins RD, Plunkett R (1980) Formulas for natural frequency and mode shape. J Appl Mech 47:461

9. Carrera E, Brischetto S, Cinefra M, Soave M (2011) Effects of thickness stretching in functionally graded plates and shells. Compos Part B Eng 42(2):123-133

10. Farsangi MA, Saidi A (2012) Levy type solution for free vibration analysis of functionally graded rectangular plates with piezoelectric layers. Smart Mater Struct 21(9):094017

11. Fesharaki JJ, Madani SG et al (2016) Effect of stiffness and thickness ratio of host plate and piezoelectric patches on reduction of the stress concentration factor. Int J Adv Struct Eng 8(3):229-242

12. Gorman $D$ (1976) Free vibration analysis of cantilever plates by the method of superposition. J Sound Vib 49(4):453-467

13. He X, Ng T, Sivashanker S, Liew K (2001) Active control of FGM plates with integrated piezoelectric sensors and actuators. Int J Solids Struct 38(9):1641-1655
14. Jadhav P, Bajoria K (2013) Free and forced vibration control of piezoelectric FGM plate subjected to electro-mechanical loading. Smart Mater Struct 22(6):065021

15. Kaczkowski Z (1968) Plates-statistical calculations. Arkady, Warsaw

16. Karama M, Afaq K, Mistou S (2003) Mechanical behaviour of laminated composite beam by the new multi-layered laminated composite structures model with transverse shear stress continuity. Int J Solids Struct 40(6):1525-1546

17. Kerr A, Alexander H (1968) An application of the extended Kantorovich method to the stress analysis of a clamped rectangular plate. Acta Mech 6(2-3):180-196

18. Kirchoff $G$ (1850) Uber das gleichgewicht und die bewegung einer elastischen scheibe. J die Reine Angew Math (Crelle's J) 40:51-88

19. Levinson M (1980) An accurate, simple theory of the statics and dynamics of elastic plates. Mech Res Commun 7(6):343-350

20. Levy M (1877) Memoire sur la theorie des plaques elastiques planes. J Math Pure Appl 219-306. http://eudml.org/doc/23515 9

21. Levy M (1899) Sur l'équilibre élastique d'une plaque rectangulaire. C R Acad Sci Paris 129:535-539

22. Liew K, Lim H, Tan M, He X (2002) Analysis of laminated composite beams and plates with piezoelectric patches using the element-free Galerkin method. Comput Mech 29(6):486-497

23. Liu G, Dai K, Lim K (2004) Static and vibration control of composite laminates integrated with piezoelectric sensors and actuators using the radial point interpolation method. Smart Mater Struct 13(6):1438

24. Liu P, Bui T, Zhu D, Yu T, Wang J, Yin S, Hirose S (2015) Buckling failure analysis of cracked functionally graded plates by a stabilized discrete shear gap extended 3-node triangular plate element. Compos Part B Eng 77:179-193

25. Loja M, Soares CM, Barbosa JI (2013) Analysis of functionally graded sandwich plate structures with piezoelectric skins, using b-spline finite strip method. Compos Struct 96:606-615

26. Mindlin R (1951) Influence of rotatory inertia and shear on flexural motions of isotropic, elastic plates. J Appl Mech 18:31-38

27. Murthy MVV (1981) An improved transverse shear deformation theory for laminated anisotropic plates. NASA Technical Paper 1903

28. Navier $L$ (1823) Extrait des recherches sur la flexion des plans elastiques. Bull Sci Soc Philomat 5:95-102

29. Nguyen TN, Thai $\mathrm{CH}$, Nguyen-Xuan H (2016) On the general framework of high order shear deformation theories for laminated composite plate structures: a novel unified approach. Int J Mech Sci 110:242-255

30. Nguyen-Quang K, Dang-Trung H, Ho-Huu V, Luong-Van $\mathrm{H}_{\text {, }}$ Nguyen-Thoi T (2017) Analysis and control of FGM plates integrated with piezoelectric sensors and actuators using cell-based smoothed discrete shear gap method (cs-dsg3). Compos Struct 165:115-129

31. Panc V (1975) Theories of elastic plates, vol 2. Springer, Berlin

32. Panda S, Ray M (2006) Nonlinear analysis of smart functionally graded plates integrated with a layer of piezoelectric fiber reinforced composite. Smart Mater Struct 15(6):1595

33. Reddy J (1984) A simple higher-order theory for laminated composite plates. J Appl Mech 51(4):745-752

34. Reddy J, Cheng ZQ (2001) Three-dimensional solutions of smart functionally graded plates. J Appl Mech 68(2):234-241

35. Reissner E (1974) On tranverse bending of plates, including the effect of transverse shear deformation

36. Rouzegar J, Abad F (2015) Free vibration analysis of FG plate with piezoelectric layers using four-variable refined plate theory. Thin Walled Struct 89:76-83 
37. Rouzegar J, Koohpeima R, Abad F (2020) Dynamic analysis of laminated composite plate integrated with a piezoelectric actuator using four-variable refined plate theory. Iran J Sci Technol Trans Mech Eng 44:557-570. https://doi.org/10.1007/s4099 7-019-00284-1

38. Sayyad A, Ghugal Y (2015) On the free vibration analysis of laminated composite and sandwich plates: a review of recent literature with some numerical results. Compos Struct 129:177-201

39. Sayyad AS, Ghugal YM (2019) A unified five-degree-of-freedom theory for the bending analysis of softcore and hardcore functionally graded sandwich beams and plates. J Sandw Struct Mater 0:1099636219840980

40. Selim B, Yin B, Liew K (2018) Impact analysis of CNT-reinforced composite plates integrated with piezoelectric layers based on Reddy's higher-order shear deformation theory. Compos Part B Eng 136:10-19

41. Senthilnathan N, Lim S, Lee K, Chow S (1987) Buckling of shear-deformable plates. AIAA J 25(9):1268-1271

42. Shakeri M, Mirzaeifar R (2009) Static and dynamic analysis of thick functionally graded plates with piezoelectric layers using layerwise finite element model. Mech Adv Mater Struct 16(8):561-575

43. Shimpi R (2002) Refined plate theory and its variants. AIAA J 40(1):137-146

44. Soldatos K (1992) A transverse shear deformation theory for homogeneous monoclinic plates. Acta Mech 94(3-4):195-220

45. Stein $M$ (1986) Nonlinear theory for plates and shells including the effects of transverse shearing. AIAA J 24(9):1537-1544

46. Szilard R (2004) Theories and applications of plate analysis: classical, numerical and engineering methods. Wiley, Hoboken

47. Thai H, Choi D (2013) Finite element formulation of various four unknown shear deformation theories for functionally graded plates. Finite Elem Anal Des 75:50-61

48. Thanh CL, Ferreira A, Wahab MA (2019a) A refined sizedependent couple stress theory for laminated composite micro-plates using isogeometric analysis. Thin Walled Struct 145:106427

49. Thanh CL, Tran LV, Vu-Huu T, Abdel-Wahab M (2019b) The sizedependent thermal bending and buckling analyses of composite laminate microplate based on new modified couple stress theory and isogeometric analysis. Comput Methods Appl Mech Eng 350:337-361
50. Tiersten H (1969) Linear piezoelectric plate vibrations. Plenum Press, New York

51. Touratier M (1991) An efficient standard plate theory. Int J Eng Sci 29(8):901-916

52. Vu TV, Khosravifard A, Hematiyan M, Bui TQ (2018) A new refined simple TSDT-based effective meshfree method for analysis of through-thickness FG plates. Appl Math Model 57:514-534

53. Wang C, Yu T, Shao G, Nguyen TT, Bui TQ (2019) Shape optimization of structures with cutouts by an efficient approach based on xiga and chaotic particle swarm optimization. Eur J Mech A Solids 74:176-187

54. Wang Q, Quek S, Sun C, Liu X (2001) Analysis of piezoelectric coupled circular plate. Smart Mater Struct 10(2):229

55. Yin S, Hale JS, Yu T, Bui TQ, Bordas SP (2014) Isogeometric locking-free plate element: a simple first order shear deformation theory for functionally graded plates. Compos Struct 118:121-138

56. Young $D$, Felgar $R$ et al (1949) Tables of characteristic functions representing nomal modes of vibration of a beam. The University of Texas, Austin

57. Yu T, Bui TQ, Yin S, Doan DH, Wu CT, Van Do T, Tanaka S (2016a) On the thermal buckling analysis of functionally graded plates with internal defects using extended isogeometric analysis. Compos Struct 136:684-695. https://doi.org/10.1016/j.comps truct.2015.11.002

58. Yu T, Yin S, Bui TQ, Xia S, Tanaka S, Hirose S (2016b) Nurbs-based isogeometric analysis of buckling and free vibration problems for laminated composites plates with complicated cutouts using a new simple fsdt theory and level set method. Thin Walled Struct 101:141-156

59. Zenkour A (2006) Generalized shear deformation theory for bending analysis of functionally graded plates. Appl Math Model 30(1):67-84

60. Zenkour A (2013a) Bending of FGM plates by a simplified fourunknown shear and normal deformations theory. Int J Appl Mech 5(02):1350020

61. Zenkour AM (2013b) A simple four-unknown refined theory for bending analysis of functionally graded plates. Appl Math Model 37(20-21):9041-9051

Publisher's Note Springer Nature remains neutral with regard to jurisdictional claims in published maps and institutional affiliations. 